ARTICLE

\title{
Microglia clear neuron-released $\alpha$-synuclein via selective autophagy and prevent neurodegeneration
}

\author{
Insup Choi ${ }^{1}$, Yuanxi Zhang ${ }^{1}$, Steven P. Seegobin (10 ${ }^{1}$, Mathilde Pruvost ${ }^{1}$, Qian Wang ${ }^{1,2}$, Kerry Purtell ${ }^{1}$,
} Bin Zhang ${ }^{2} \&$ Zhenyu Yue ${ }^{1 凶}$

Microglia maintain brain homeostasis by removing neuron-derived components such as myelin and cell debris. The evidence linking microglia to neurodegenerative diseases is growing; however, the precise mechanisms remain poorly understood. Herein, we report a neuroprotective role for microglia in the clearance of neuron-released $\alpha$-synuclein. Neuronal $\alpha$-synuclein activates microglia, which in turn engulf $\alpha$-synuclein into autophagosomes for degradation via selective autophagy (termed synucleinphagy). Synucleinphagy requires the presence of microglial Toll-like receptor 4 (TLR4), which induces transcriptional upregulation of p62/SQSTM1 through the NF- $\mathrm{kB}$ signaling pathway. Induction of p62, an autophagy receptor, is necessary for the formation of $\alpha$-synuclein/ubiquitin-positive puncta that are degraded by autophagy. Finally, disruption of microglial autophagy in mice expressing human $\alpha$-synuclein promotes the accumulation of misfolded $\alpha$-synuclein and causes midbrain dopaminergic neuron degeneration. Our study thus identifies a neuroprotective function of microglia in the clearance of $\alpha$-synuclein via TLR4-NF-kB-p62 mediated synucleinphagy.

\footnotetext{
${ }^{1}$ Departments of Neurology and Neuroscience, Friedman Brain Institute, Icahn School of Medicine at Mount Sinai, New York, NY 10029, USA. ${ }^{2}$ Department of Genetics and Genomic Sciences, Icahn Institute of Genomics and Multiscale Biology, Icahn School of Medicine at Mount Sinai, New York, NY 10029, USA.

凶email: zhenyu.yue@mssm.edu
} 
-synuclein is produced primarily in neurons and constitutes up to $1 \%$ of total cytosolic protein in the brain ${ }^{1}$. Although the function of $\alpha$-synuclein is poorly understood, available evidence suggests its role in synaptic vesicular trafficking ${ }^{2-4}$. Aggregated $a$-synuclein is a major component in intraneuronal inclusions known as Lewy bodies (LB) associated with neurodegenerative diseases, such as Parkinson's disease (PD) and dementia with Lewy body (DLB) $)^{5,6}$. Increased $\alpha$-synuclein level due to the multiplication of SNCA alleles is causal to $\mathrm{PD}^{7,8}$.

Previous evidence suggested "prion-like" cell-to-cell transmission of $\alpha$-synuclein ${ }^{9,10}$. $\alpha$-synuclein can be secreted by neurons as a result of cellular stress or injury, or as a response to stimulation ${ }^{11-13}$. Neighboring neurons and glia can engulf and clear extracellular $\alpha$-synuclein and thus contributing to the regulation of $\alpha$-synuclein homeostasis in the brain. Interestingly, injection of fibrillar $a$-synuclein into animal brains causes the spread of LBlike pathology ${ }^{14}$, supporting the "Braak hypothesis" of staging in synucleinopathies ${ }^{15}$. Therefore, cellular uptake and clearance pathways are key processes to control the deposition and spread of a-synuclein aggregates, thus affecting disease progression.

Although neurons and glia in the brain can ingest and degrade extracellular a-synuclein, microglia show the highest efficiency in vitro ${ }^{16}$. Extensive effort has been made toward the identification of cellular pathways of $a$-synuclein clearance. Several studies reported receptor-mediated internalization of various forms of $\alpha$ synuclein in neurons and glial cells ${ }^{17-19}$. However, the exact mechanism for the clearance of internalized $\alpha$-synuclein remains unclear.

Previous studies suggest that $a$-synuclein is degraded by macroautophagy, chaperone-mediated autophagy, and the proteasome $\mathrm{e}^{20-23}$. However, the evidence for macroautophagy (hereafter referred to as autophagy) degradation of a-synuclein is extremely limited ${ }^{24,25}$. Autophagy is a bulk degradation pathway responsible for the clearance of protein aggregates and damaged cellular organelles ${ }^{26,27}$. Recent evidence demonstrates a strong selectivity for autophagy ${ }^{28}$. Direct evidence for autophagy in selective degradation of $a$-synuclein is entirely lacking. Furthermore, since most of the studies of a-synuclein degradation were focused on neurons, whether or not microglia, the prototypical scavenger cell in the brain, take part in the degradation of $\alpha$ synuclein remains unclear.

Here, we report that microglia ingest and degrade neuronreleased a-synuclein through selective autophagy in vitro and in vivo. We document that ingested $\alpha$-synuclein in microglia is sequestered by autophagosomes for degradation, which is mediated by TLR4-NF- $\mathrm{kB}$ signaling through upregulation of the autophagy receptor, $p 62 / S Q S T M 1$. Thus, our results uncover a microglia-specific a-synuclein degradation pathway termed synucleinphagy that regulates a-synuclein homeostasis in the CNS.

\section{Results}

Neuron-released a-synuclein is engulfed by microglia in vivo. To understand microglia and $a$-synuclein interaction in vivo, we employed two mouse models, both of which express wild-type (WT) human $\alpha$-synuclein (ha-Syn) in a neuron-specific manner. We first verified that mice injected with adeno-associated virus 9 (AAV9) carrying green fluorescent protein (GFP) under the synapsin promoter into the Substantia Nigra pars compacta (SNpc) expressed GFP strictly in neurons (Supplementary Fig. 1a, b). Mice injected with AAV9-ha-Syn in the SNpc showed loss of dopaminergic neurons on the ipsilateral side (Supplementary Fig. 1c), as reported ${ }^{29}$. In fact, dopamine transporter (DAT) and tyrosine hydroxylase $(\mathrm{TH})$ protein levels were reduced compared with AAV9-GFP-injected mice (Supplementary Fig. 1d, e).
Moreover, AAV9-ha-Syn, but not AAV9-GFP injection, caused microglial activation in the striatum as evidenced by the reduced length of processes and terminal branch points as well as increased cell number (Fig. 1a, b).

By RT-qPCR analysis of microglia state-specific gene expres$\operatorname{sion}^{30-32}$, we found upregulation of several disease-associated signature genes such as $A p o E, A x l$, and $L p l$, inflammatory gene Tnf, and Tgfbrl at 4-weeks post AAV injection (Fig. 1c; Supplementary Fig. 2). Importantly, we verified the presence of $h a-S y n$ protein in isolated microglia via western blot with haSyn-specific antibody (Fig. 1d; Supplementary Fig. 3a). Using Cx3cr1 CreER-IRES-Eyfp transgenic mice ${ }^{33}$, which express enhanced yellow fluorescent protein (EYFP) under the microglia-specific Cx3crl promoter, we detected ha-Syn in Cx3cr1-EYFP-positive microglia (Fig. 1e; Supplementary Fig. 3b).

In transgenic mice overexpressing ha-Syn under the Thy-1 promoter (ha-Syn-Tg), we also found microglial activation in the striatum of 10-month-old mice (Fig. 1f, g). ha-Syn protein was clearly present in microglia through western blot analysis (Fig. 1h) and immunofluorescent staining in Iba-1-positive microglia at the striatum (Fig. 1i; Supplementary Fig. 3c). Noticeably, ha-Syn-Tg mice did not show degeneration of dopaminergic neurons in SNpc up to 12-month-old (Supplementary Fig. 1f). Together, our data from two different mouse models demonstrate that neuronreleased ha-Syn induces microglial activation, accompanied by microglial engulfment of extracellular ha-Syn in vivo.

Microglia-engulfed $\alpha$-synuclein is degraded by autophagy. We next asked if ingested $\alpha$-synuclein goes through endocytic degradation following phagocytosis or receptor-mediated endocytosis $^{18,19}$. We treated cultured primary microglia with recombinant ha-Syn protein and found no evidence of colocalization between ha-Syn and an early endosome marker EEA1 (Supplementary Figs. 3d, 4a). Neither cytochalasin D, a blocker of actin polymerization necessary for phagocytosis, nor Dynasore, a GTPase inhibitor that suppresses both clathrin-dependent and independent endocytosis, inhibited the uptake of ha-Syn protein but rather it increased the uptake (Supplementary Figs. 4b, 5a, 5b).

We then asked whether autophagy degrades ha-Syn in microglia. We co-stained ha-Syn-treated primary microglia with anti-ha-Syn and anti-ubiquitin or anti-p62/SQSTM1, an autophagy receptor $^{34}$, and observed colocalization of ha-Syn with ubiquitin or p62 in discrete puncta (middle panels, Fig. 2a; left panels, Fig. 2b). We also treated microglia derived from GFP-LC3 transgenic mice ${ }^{35}$ with $h a-S y n$ and detected colocalization of the autophagosome marker, GFP-LC3, and ha-Syn (right panels, Fig. 2b). These ha-Syn-associated ubiquitin-, GFP-LC3-, or p62positive puncta appeared at $6 \mathrm{~h}$ and disappeared at $24 \mathrm{~h}$ after $h a-$ Syn treatment (arrowheads, Fig. 2a). We also noticed smaller ubiquitin-negative ha-Syn particles that were evident after $6 \mathrm{~h}$, but remained $24 \mathrm{~h}$ after treatment (arrows, Fig. 2a). A time-course

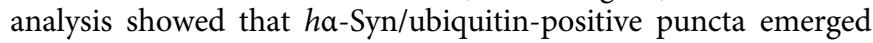
at $3 \mathrm{~h}$, and the number of puncta peaked at $6 \mathrm{~h}$, followed by a reduction in number at $24 \mathrm{~h}$ post treatment (left panel, Fig. 2c). The puncta number also depended on ha-Syn concentration (right panel, Fig. 2c). In addition, we also observed ha-Syn-associated ubiquitin-, GFP-LC3-positive puncta after treating the medium from AAV9-ha-Syn-infected cortical neuron (arrowheads, Supplementary Fig. 4c), suggesting that ha-Syn-associated LC3 puncta also occurs in microglia in response to physiological form of a-Syn. Furthermore, in GFP-LC3 transgenic mice injected with AAV9ha-Syn, we found ingested ha-Syn colocalized with GFP-LC3 puncta in Iba-1-positive microglia in the striatum (Fig. 2d). We then performed ultrastructural analysis through electron microscopy (EM) and found that ha-Syn-treated microglia develop large 
a

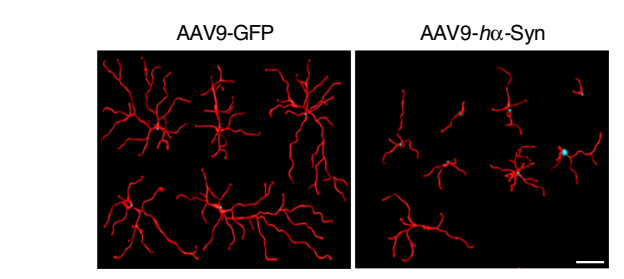

d

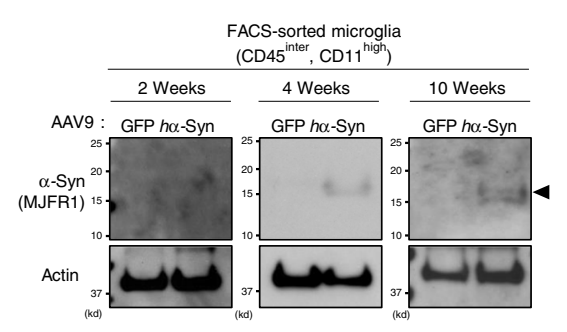

b

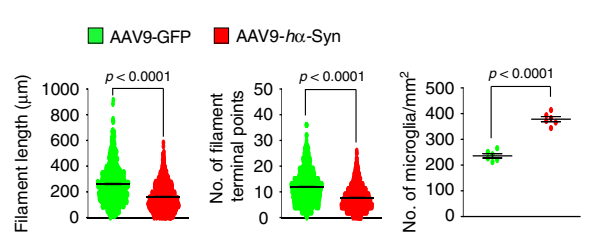

e

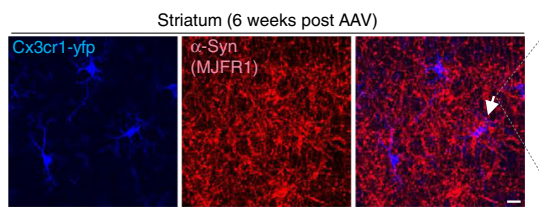

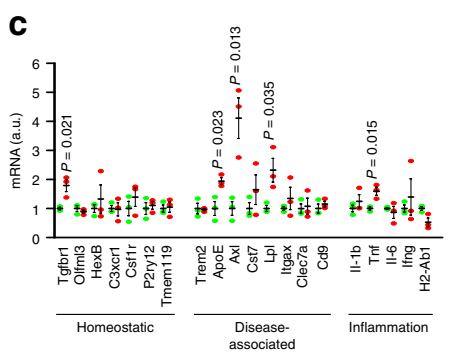

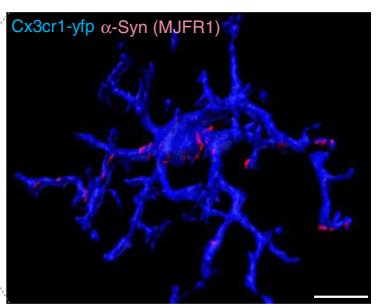

f
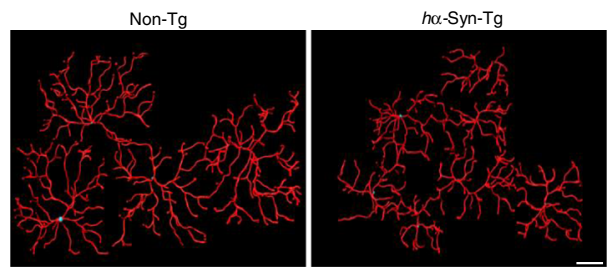

$\mathbf{h}$
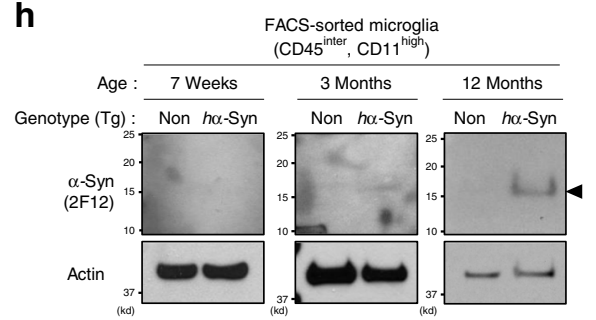

i g

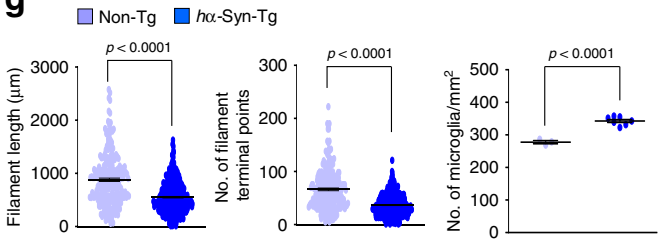

Fig. 1 Neuron-released $\boldsymbol{\alpha}$-synuclein is engulfed by microglia in vivo. $\mathbf{a}, \mathbf{b}, \mathbf{f}, \mathbf{g}$ Brain sections from AAV-GFP $(n=768$ cells, six animals) and AAV-h $\alpha$-Syninjected mice ( $n=986$ cells, six animals) at 6 weeks post AAV injection (a, b) or from 10-month-old Non- $\operatorname{Tg}(n=246$ cells, three animals) and ho-Syn-Tg $(n=478$ cells, seven animals) mice $(\mathbf{f}, \mathbf{g})$ were stained for a marker of microglia, Iba-1, to visualize microglia morphology in the striatum. Reconstruction of microglia processes was generated by Filament-Tracer tool in Imaris software $(\mathbf{a}, \mathbf{e})$ and filament lengths $(p<0.0001)$ and the number of terminal points $(p<0.0001)$ were quantified (left panels in $\mathbf{b}$ and $\mathbf{g}$ ). The number of microglia at the striatum was counted using Image J software (NIH, Bethesda, MD) (right panel in $\mathbf{b}(p=6.59 \mathrm{E}-07)$ and $\mathrm{g}(p=4.88 \mathrm{E}-05))$. $p$-values were calculated by two-tailed Mann-Whitney $U$ test (the left two panels) and unpaired two-tailed Student's $t$ test (the right panel). Scale bar, $10 \mu \mathrm{m}$. c CD45 intermediate and CD11b high microglia were isolated from brains injected with AAV-h $\alpha$ Syn $(n=3)$ and assayed for RT-qPCR using primers as indicated, compared with AAV-GFP $(n=3)$ at 4-weeks after AAV. p-values were calculated by unpaired two-tailed Student's $t$ test. d, h Pooled CD45intermediate and CD11bhigh microglia were prepared either from AAV-GFP ( $n=3$ ) and AAV-h $\alpha$-Syninjected mice $(n=3)$ (d) or Non-Tg $(n=4)$ and $h \alpha-S y n-\operatorname{Tg}(n=4)$ mice $(\mathbf{h})$ brains at the indicated time points and lysed for W.B using $\alpha$-synuclein antibodies (MJFR1 clone in d, 2F12 clone in $\mathbf{h}$ ) and actin. e, i Brain sections from Cx3cr7CreER-IRES-Eyfp mice injected with AAV-h $\alpha$-Syn were stained using anti-GFP/YFP antibody and human $\alpha$-synuclein antibody at 6 weeks after AAV (e), and brain sections from 10-month-old Non-Tg and $\alpha$-Syn-Tg mice were stained using Iba-1 antibody and human $\alpha$-Synuclein antibody (i). 3D reconstruction of microglia and human $\alpha$-synuclein was produced using Imaris software (Bitplane, right panel) as described in the "Methods" section. Scale bar, $10 \mu \mathrm{m}$. All values are reported as mean \pm SEM. Data are representative of three independent experiments.

inclusions which are surrounded by double-membrane structures, characteristic of autophagosomes (Fig. 2e).

We next treated microglia with SAR405, an inhibitor of VPS34, which is a class III phosphatidylinositol 3-kinase required for autophagosome membrane synthesis ${ }^{36}$, Bafilomycin A1, or Chloroquine, two different inhibitors of lysosome acidification, after adding ha-Syn. All three drugs prevented the clearance of ha-Syn/ubiquitin-positive puncta (Fig. 2f). By contrast, treatment with Torin1, an inhibitor of mTOR that stimulates autophagy ${ }^{37}$, facilitated the clearance of puncta (Fig. 2g). We next obtained autophagy-deficient microglia by breeding $\mathrm{C} x 3 \mathrm{cr} 1^{\mathrm{Cre}}$ mice, which constitutively express Cre recombinase, with either Atg $7^{\text {flox/flox }}$ or
Atg14 flox/flox mice. Atg7 encodes an E1-like enzyme essential in the ubiquitin-like conjugation systems required for autophagy, and Atg14 encodes a positive regulator of VPS34 (refs. ${ }^{38,39}$ ). The lack of autophagy was confirmed in Atg7-deficient cells or Atg14deficient cells through the analysis of Atg7, Atg14, p62, and LC3II (Fig. 2h). In these autophagy-deficient microglia, degradation of ha-Syn/ubiquitin-positive puncta was inhibited (Fig. 2i). We noticed that Atg7 or Atg14 deficiency did not cause microglia death regardless of ha-Syn protein treatment (Supplementary Fig. 4d, e). Thus, we concluded that microglia engulf extracellular a-syn protein, which is sequestered by autophagosomes and degraded by the autophagy-lysosome pathway. 
a

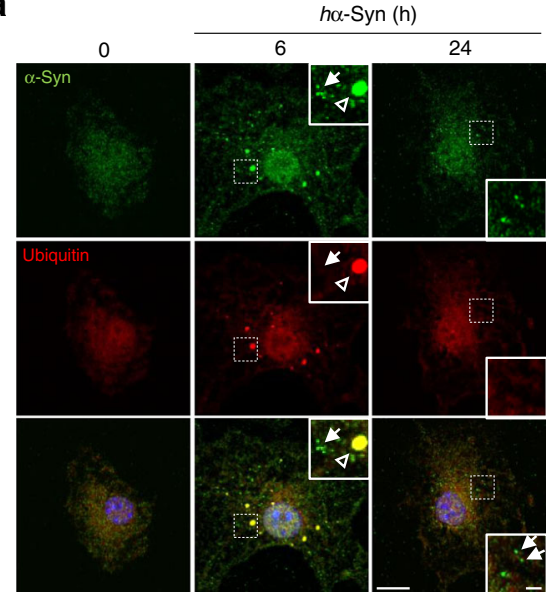

e

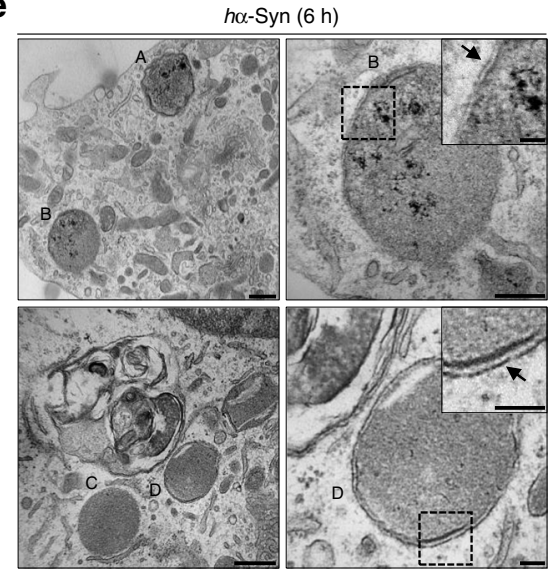

b

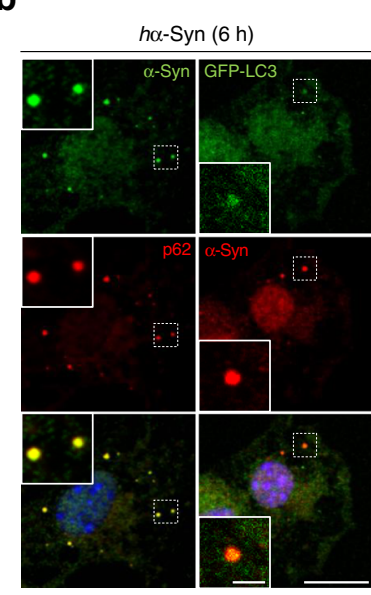

$\mathbf{f}$
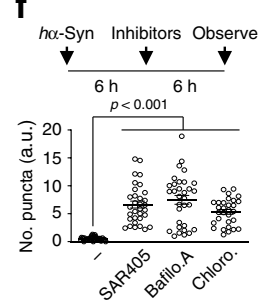

g

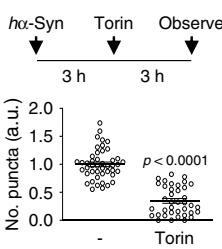

h
C
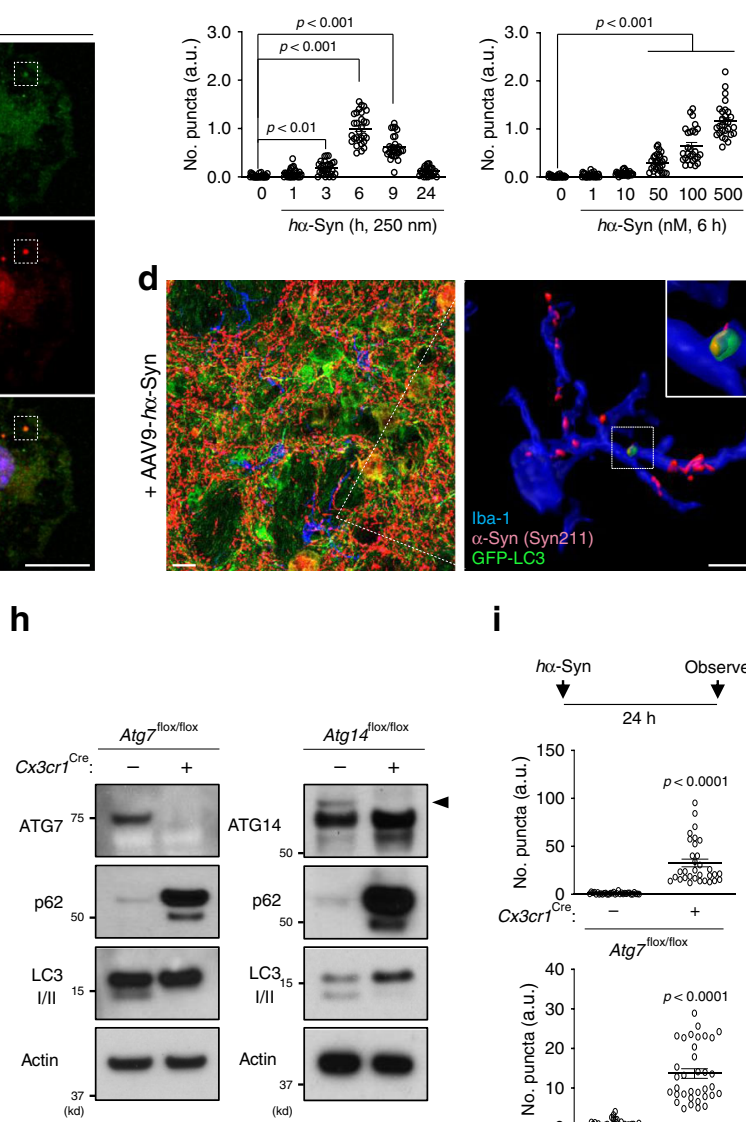

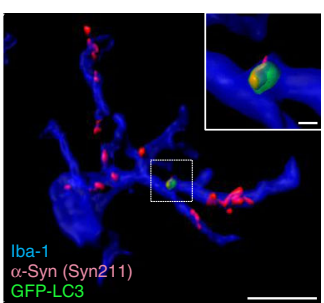

i
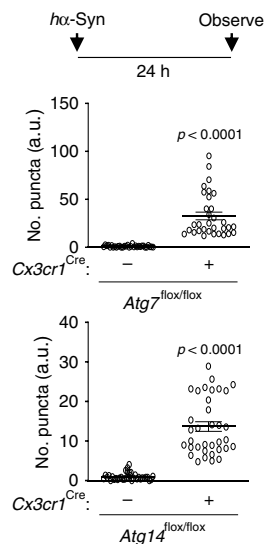

Fig. 2 Microglia-engulfed $\boldsymbol{\alpha}$-synuclein is degraded by autophagy. $\mathbf{a}, \mathbf{b}, \mathbf{c}$ Cultured primary microglia from WT mice (a and left panels of $\mathbf{b}$ ) and GFP-LC3transgenic mice (right panels of $\mathbf{b}$ ) were treated $250 \mathrm{nM} h \alpha-S y n$ protein and stained with antibodies against human $\alpha$-synuclein (MJFR1 clone, a and $\mathbf{b}$ ), ubiquitin (a), or p62 (b). The number of $h \alpha$-Syn/ubiquitin-positive puncta was quantified (c, $n=29$, three independent experiments). In a, empty arrowheads indicate a portion of $\alpha$-synuclein colocalized with ubiquitin-positive puncta and disappear in $24 \mathrm{~h}$. Arrows represent a portion of $\alpha$-synuclein not colocalized with ubiquitin. $p$-values were calculated by one-way ANOVA with Newman-Keuls post hoc test. $F(5,168)=139.6, p=6.43 E-58$, left panel; $F(5,162)=118.4, p=3.14 \mathrm{E}-52$. Scale bar, $10 \mu \mathrm{m} ; 2 \mu \mathrm{m}$ in magnified boxes. $\mathbf{d}$ At 6 weeks post AAV-h $\alpha$-Syn inoculation into GFP-LC3 mice, brain slices were fixed and stained with antibodies against human $\alpha$-synuclein, GFP, and lba-1 (left panel). 3D surface-rendering images of each protein in microglia were made with Imaris software (Bitplane, right panel). Scale bar, $10 \mu \mathrm{m} ; 1 \mu \mathrm{m}$ in the magnified box. e Ultrastructure of puncta from $250 \mathrm{nM}$ h $\alpha$-Syn proteintreated cells for $6 \mathrm{~h}$ was observed by an electron microscope. Arrows indicate double-membrane structures. Scale bar, $500 \mathrm{~nm}$ in the left panel; $100 \mathrm{~nm}$ in the right panel. $\mathbf{f}, \mathbf{g}$ Microglia were treated with $250 \mathrm{nM} h \alpha$-Syn protein and SAR405 (ff, $n=32)$, Bafilomycin A1 (f, $n=32)$, and Chloroquine (f, $n=30)$ or Torin $(\mathbf{g}, n=38)$ was added. The number of $h \alpha$-Syn/ubiquitin-positive puncta was quantified in the lower panel. $p$-values were calculated by one-way ANOVA with Newman-Keuls post hoc test $(\mathbf{f}, \mathrm{F}(3,130)=39.88, p=2.43 \mathrm{E}-18)$ and unpaired two-tailed Student's $t$ test $(\mathbf{g}, p=5.38 \mathrm{E}-18)$. $\mathbf{h}, \mathbf{i}$ Microglia cultured either from Atgflox/flox mice (left panel) from Atg14flox/flox mice (right panel) with or without Cx3cr7Cre expression were assayed for W.B using antibodies against ATG7 or ATG14, p62, and LC3 I/II (h). Cells were treated with $250 \mathrm{nM}$ h $\alpha$-Syn protein for $24 \mathrm{~h}$, and the number of ho-Syn/ubiquitinpositive puncta was quantified ( $\mathbf{I}, n=32, p<0.0001$ for Atg7; $n=35, p<0.0001$ for Atg14). $p$-values were calculated by two-tailed Mann-Whitney $U$ test. All values are reported as mean \pm SEM. Data are representative of at least three independent experiments.

p62 mediates sequestration and degradation of a-synuclein. p62 is known to recognize and interact with ubiquitinated cargo to initiate selective autophagy 34 . We next investigated the role of p62 in autophagic clearance of ha-Syn. We observed that ha-Syn treatment caused an increase in p62 protein up to $9 \mathrm{~h}$ (Fig. $3 \mathrm{a}$ ). Interestingly, $p 62 \mathrm{mRNA}$ levels peaked at $3 \mathrm{~h}$, declined at $6 \mathrm{~h}$, and returned to baseline at $9 \mathrm{~h}$ post treatment (Fig. $3 \mathrm{~b}$ ), which mirrors the transient increase of ha-Syn/ubiquitin/p62/GFP-LC3-positive puncta (Fig. 2a-c). Actinomycin D, a blocker of mRNA translation, inhibited p62 induction when added before ha-Syn treatment (Supplementary Fig. 4g) and suppressed the formation of ha-Syn/ubiquitin-positive puncta (Supplementary fig. 4h). By contrast, total LC3-II levels changed little after ha-Syn treatment (Supplementary Fig. 4i), suggesting no significant alteration of overall autophagy. Furthermore, other autophagy receptors such as Ndp52, Optineurin, and Nbr1 were not altered (Supplementary Fig. 4i), suggesting that ha-Syn selectively induces p62 expression without affecting other autophagy receptors.

We next treated cultured microglia from $p 62-\mathrm{KO}$ mice with ha-Syn (Fig. 3c). Remarkably, ha-Syn/ubiquitin-positive puncta were abolished in p62-KO cells (Fig. 3d, e); meanwhile, p62 deficiency did not cause cell death regardless of ha-Syn treatment (Supplementary Fig. 4f), suggesting that p62 is necessary for the formation of ha-Syn/ubiquitin-positive puncta after ha-Syn ingestion, consistent with its role in selective autophagy ${ }^{34}$. In agreement with the in vitro results (Fig. 3a, b), the number of p62 puncta in microglia was significantly increased in the striatum of AAV9-ha-Syn-injected mice at 6 weeks post injection (Fig. 3f, g) 
a

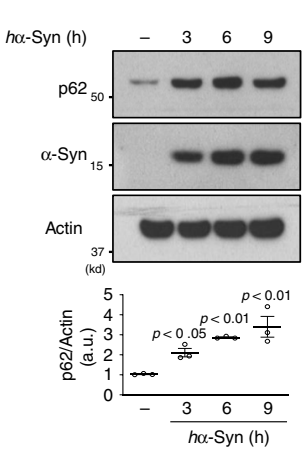

b

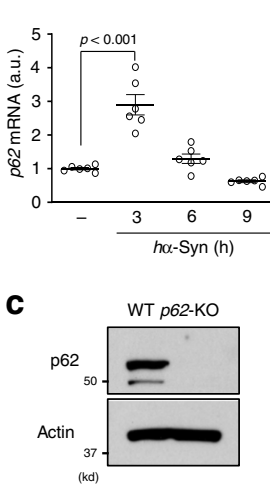

d

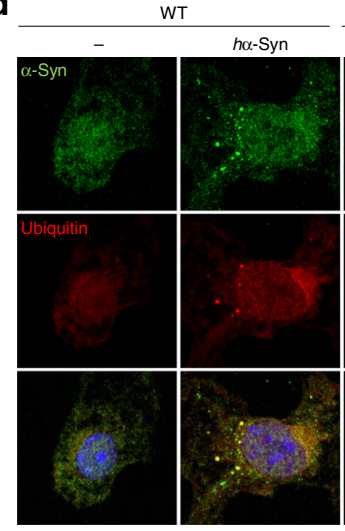

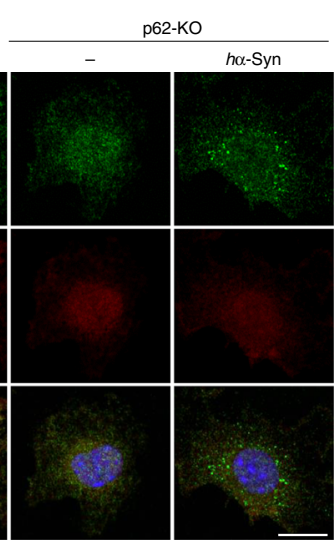

e

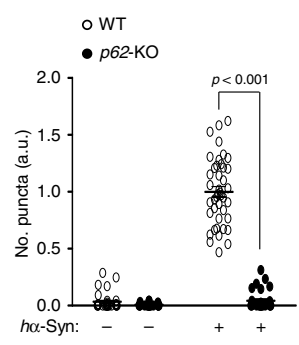

$\mathbf{f}$
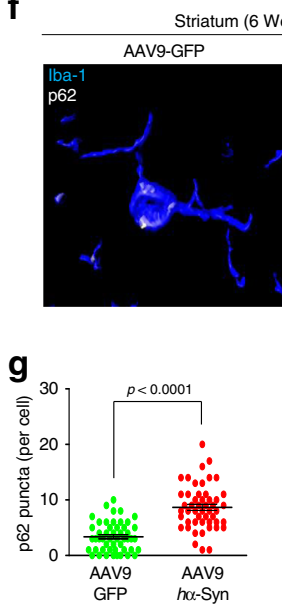
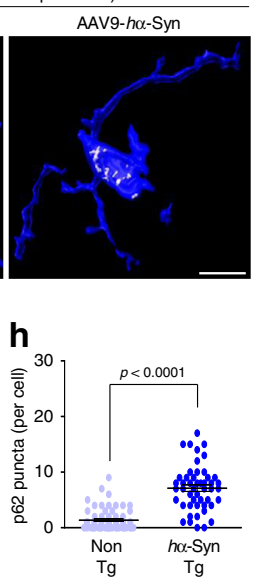

i

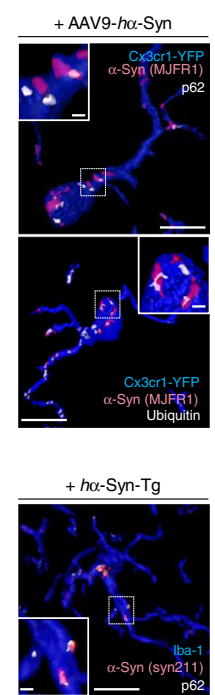

k

K AAV9-ho-Syn-injected brain I

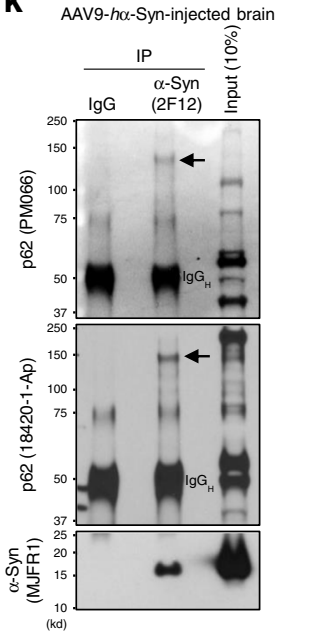

$h \alpha-$ Syn-Tg brain

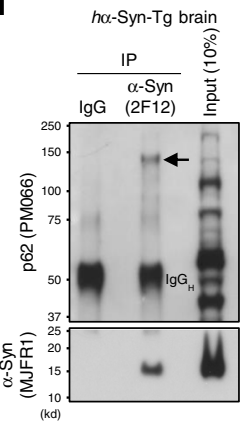

m

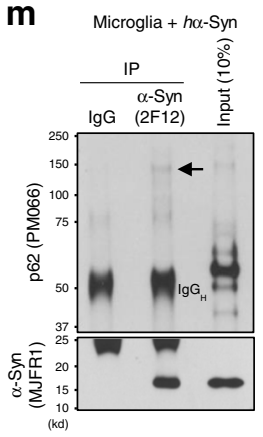

Fig. 3 p62 mediates sequestration and degradation of $\boldsymbol{\alpha}$-synuclein. a Microglia were treated with $250 \mathrm{nM}$ h $\alpha$-Syn protein for the indicated time and assayed for W.B using antibodies against p62 and human $\alpha$-synuclein. The levels of p62 protein were quantified in the lower panel. $n=3$ over three independent experiments. $p$-values were calculated by one-way ANOVA with Newman-Keuls post hoc test. $F(3,8)=13.44, p=0.0017$. b After treatment of $250 \mathrm{nM} h \alpha$-Syn protein for the indicated time, the levels of $p 62$ mRNA were examined by RT-qPCR. p62 mRNA was normalized to Actin mRNA. $n=6$ over three independent experiments. $p$-values were calculated by one-way ANOVA with Newman-Keuls post hoc test. $F(3,20)=35.43, p=3.39 \mathrm{E}-08$. c The absence of $\mathrm{p} 62$ protein was confirmed in microglia obtained from p62-KO mice by W.B. d, e WT and p62-KO microglia were treated with $250 \mathrm{nM}$ h $\alpha$ Syn protein for $6 \mathrm{~h}$, and stained with antibodies against human $\alpha$-synuclein and ubiquitin (d). The number of $h \alpha$-Syn/ubiquitin-positive puncta was quantified (e). $p$-values were calculated by two-way ANOVA with Bonferroni post hoc test. $\mathrm{F}(1,139)=272.5, p=1.44 \mathrm{E}-34$ for h $\alpha$-Syn-genotype interaction; $\mathrm{F}(1,139)=315.3, p=1.45 \mathrm{E}-37$ for h $\alpha$-Syn; $F(1,139)=303.7, p=8.81 \mathrm{E}-37$ for genotype. Scale bar, $10 \mu \mathrm{m}$. $\mathbf{f}-\mathbf{h}$ The number of p62 puncta was quantified in microglia at striatum of brains either from AAV-h $\alpha$-Syn-injected mice $(n=3, \mathbf{f}, \mathbf{g}, p<0.0001)$ or from ho-Syn-Tg mice $(n=5, \mathbf{h}, p<0.0001)$ compared with AAV-GFP-injected mice $(n=3)$ and non-Tg mice $(n=3)$, respectively. $p$-values were calculated by two-tailed Mann-Whitney $U$ test. Scale bar, $10 \mu$ m. i At 6 weeks post AAV-h $\alpha$-Syn administration into Cx3cr7CreER-IRES-Eyfp mice, brain slices were stained with antibodies against GFP/YFP, human $\alpha$-synuclein, p62 (left panel), and ubiquitin (right panel). Scale bar, $10 \mu \mathrm{m} ; 1 \mu \mathrm{m}$ in the magnified box. j Brain sections from 10-month-old ho-syn-Tg mice were fixed and stained using antibodies against human $\alpha$-synuclein, Iba-1, and p62. Scale bar, $10 \mu \mathrm{m} ; 1 \mu \mathrm{m}$ in the magnified box. $\mathbf{k}, \mathbf{I}$, $\mathbf{m}$ Human $\alpha$-synuclein were immunoprecipitated from AAV-h $\alpha$-Syn-injected brain (k), h $\alpha$-Syn-Tg brain (I), and cultured microglia treated with $250 \mathrm{nM} h \alpha$-Syn protein for $6 \mathrm{~h}$ ( $\mathbf{m}$ ). Arrows indicate the high molecular weight of p62. Data are representative of at least three independent experiments. All values are reported as mean \pm SEM.

and in 10-month-old ha-Syn-Tg mice compared with controls (Fig. 3h). Furthermore, p62/ubiquitin and ha-Syn puncta colocalized in EYFP-positive (AAV9-ha-Syn-injected C $x 3$ cr 1 CreER-IRES-Eyfp) or Iba-1-positive (ha-Syn-Tg mice) microglia (Fig. 3i, j). Through co-immunoprecipitation analysis, we found that $h a-S y n$ interacted with specific p62 protein species of high molecular weight in AAV9-ha-Syn-injected brain, ha-SynTg brain, and primary microglia treated with ha-Syn (Fig. $3 \mathrm{k}-\mathrm{m}$ ), supporting that oligomeric p62 directly binds and recruits $h a-S y n$ into autophagosomes. The above data thus demonstrates that haSyn-induced p62 upregulation in microglia confers a selective autophagic degradation of ingested ha-Syn. a-synuclein-induced p62 upregulation requires TLR4. We next asked how microglia upregulate $p 62$ transcription in response to extracellular ha-Syn. Previous reports showed a-synuclein can trigger TLR4 or TLR2 activation in cultured glia, which leads to an increase in inflammatory gene transcription ${ }^{19,40,41}$. To test TLR4 involvement, we treated microglia from Tlr4-KO mice with ha-Syn and observed no increase in p62 protein and mRNA regardless of the dose (Fig. $4 \mathrm{a}, \mathrm{b}$ ). Also, the number of ha-Syn/ ubiquitin-positive puncta present at $6 \mathrm{~h}$ after ha-Syn treatment was significantly decreased in Tlr4-KO microglia compared with control cells (Fig. 4c). In addition, we treated cultures with TAK242, an inhibitor of TLR4 signaling by selectively binding to 
a
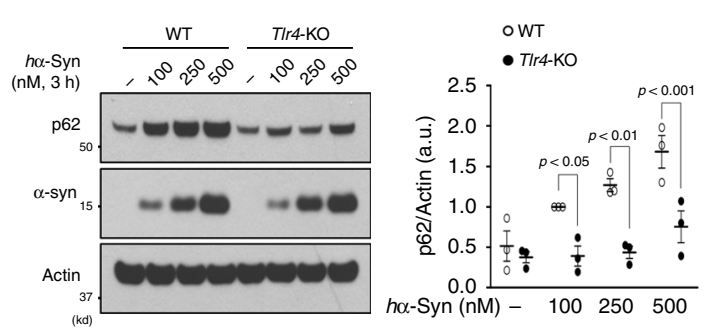

b

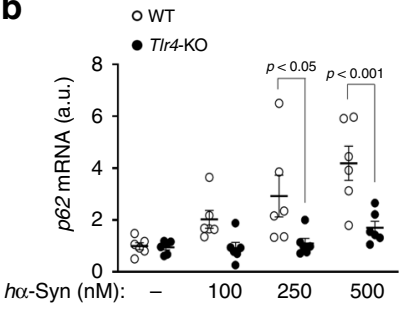

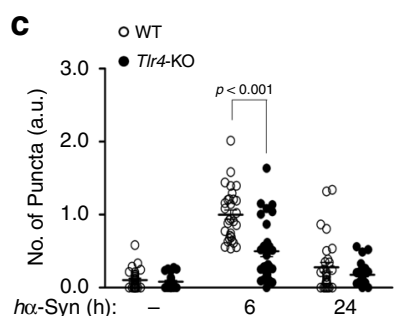

d

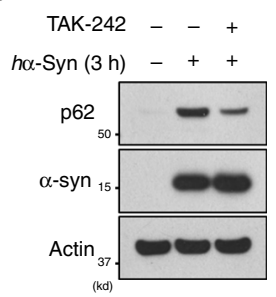

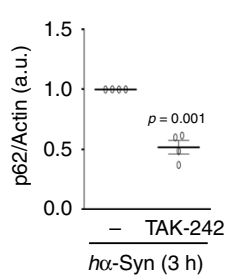

e

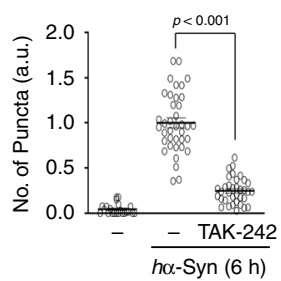

f

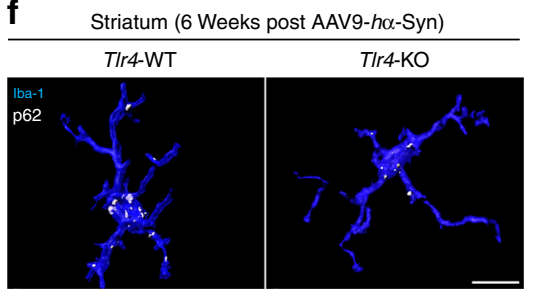

g

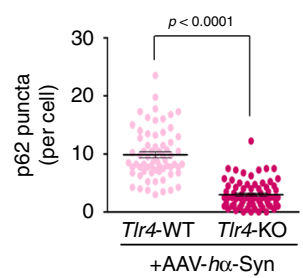

h

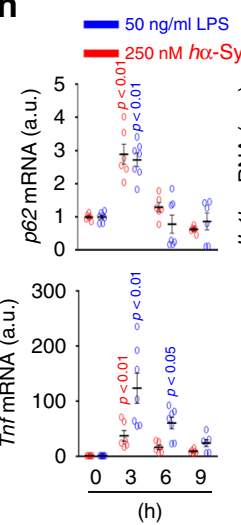

i

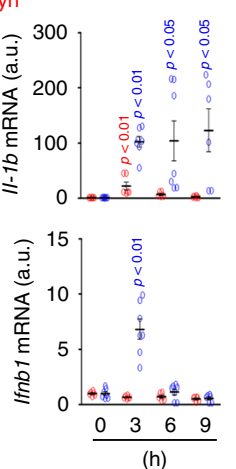

j

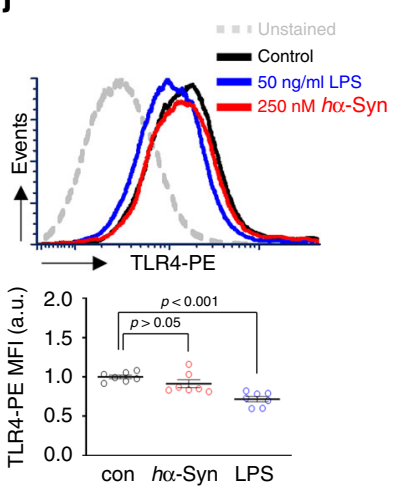

$\mathbf{k}$

Dynasore $(\mu \mathrm{M}) \quad-\quad-2050$ ho-Syn (3 h) - + + +
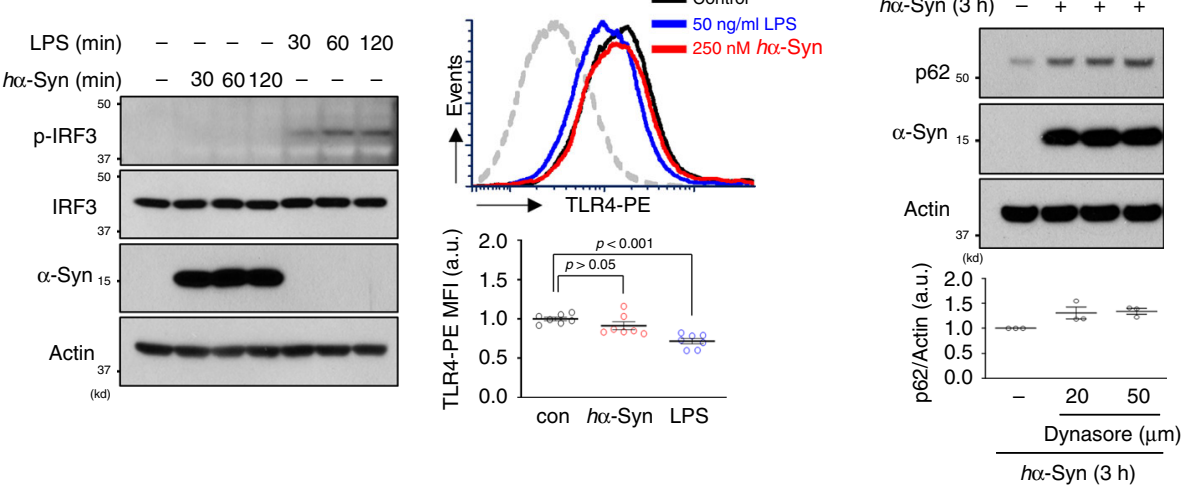

Fig. $4 \alpha$-synuclein-induced $\mathbf{p 6 2}$ upregulation requires TLR4. a, b, c Microglia obtained from WT mice and T/r4-KO mice were treated with h $\alpha$-Syn protein and assayed for W.B $(\mathbf{a}, n=3)$, RT-qPCR $(\mathbf{b}, n=6)$ or for immunostaining $(\mathbf{c}, n=30)$. Band intensities were quantified in the right panel of $\mathbf{a}$. The number of $h \alpha$-Syn/ubiquitin-positive puncta was quantified (c). $p$-values were calculated by two-way ANOVA with Bonferroni post hoc test. In $(\mathbf{a}), \mathrm{F}(3,16)=3.381$, $p=0.0443$ for h $\alpha$-Syn-genotype interaction; $F(3,6)=11.46, p=0.0003$ for h $\alpha$-Syn; $F(1,16)=43.09, p=6.51 E-06$ for genotype. In $\mathbf{b}, F(3,40)=3.199, p=$ 0.0334 for h $\alpha$-Syn-genotype interaction; $F(3,40)=8.326, p=0.0002$ for h $\alpha$-Syn; $F(1,40)=21.77, p=3.41 E-05$ for genotype. In $\mathbf{c}, F(2,174)=12.56, p=$ 8.03E-06 for h $\alpha$-Syn-genotype interaction; $F(2,174)=91.61, p=6.64 E-28$ for $h \alpha-S y n ; F(1,174)=24.89, p=1.46 E-06$ for genotype. d, e Cells were pretreated with TAK-242 and treated with $250 \mathrm{nM} h \alpha$-Syn protein. The protein levels of p62 and the number of ho-Syn/ubiquitin-positive puncta were determined by W.B $(\mathbf{d}, n=4)$ and immunostaining $(\mathbf{e}, n=35)$, respectively. $p$-values were calculated by unpaired two-tailed Student's $t$ test $(\mathbf{d}, p=$ $0.0001)$ and one-way ANOVA with Newman-Keuls post hoc test $(\mathbf{e}, F(2,92)=156.7, p=2.35 \mathrm{E}-30)$. f Representative 3D reconstruction pictures of microglia containing p62 in WT and TIr4-KO mice injected with AAV-h $\alpha$-Syn brains. Scale bar, $10 \mu \mathrm{m}$. $\mathbf{g}$ The number of p62 puncta was quantified in microglia at striatum of WT mice $(n=5)$ and T/r4-KO mice $(n=5)$. $p$-values were calculated by two-tailed Mann-Whitney $U$ test. $\mathbf{h}$ Microglia were treated with either $250 \mathrm{nM} \mathrm{h \alpha}$-Syn protein (red, $n=6$ ) or $50 \mathrm{ng} / \mathrm{ml}$ LPS (blue, $n=7$ for $0-6 \mathrm{~h}, n=6$ for $9 \mathrm{~h}$ ) for indicated time and assayed for RT-qPCR. Each mRNA level was normalized to Actin mRNA. $p$-values were calculated by one-way ANOVA with Newman-Keuls post hoc test. In h $\alpha$-Syn protein treatment, $F(3,20)=35.43, p=3.39 \mathrm{E}-08$ for $p 62, \mathrm{~F}(3,20)=6.655, p=0.0027$ for $11-1 b, \mathrm{~F}(3,20)=8.907, p=0.0006$ for $\operatorname{Tnf}, \mathrm{F}(3,20)=5.104, p=0.0087$ for Infb1; In LPS treatment, $\mathrm{F}(3,23)=19.53, p=1.61 \mathrm{E}-06$ for $p 62, \mathrm{~F}(3,23)=4.643, p=0.0111$ for $I 1-1 b, \mathrm{~F}(3,23)=12.09, p=5.92 \mathrm{E}-05$ for Tnf, $\mathrm{F}(3,23)=35.86, p=7.68 \mathrm{E}-$ 09 for Infb1. i After treating $250 \mathrm{nM}$ ho-Syn protein or $50 \mathrm{ng} / \mathrm{ml}$ LPS for indicated time, cells were assayed for W.B using antibodies against p-IRF3 (S396), IRF3, and $\alpha$-synuclein. j After treating h $\alpha$-Syn protein $(n=7)$ or LPS $(n=7)$ for $30 \mathrm{~min}$ to microglia, the level of TLR4 receptor endocytosis was determined by detecting surface TLR4 using flow cytometry. $p$-values were calculated by one-way ANOVA with Newman-Keuls post hoc test. F $(2,18)=15.10, p=$ 0.0001. k Microglia were pretreated with Dynasore $(n=3)$ for indicated concentration and treated with $250 \mathrm{nM} h \alpha-S y n$ protein for $3 \mathrm{~h}$. Data are representative of at least three independent experiments. All values are reported as mean \pm SEM.

Cys747 of TLR4 cytoplasmic tail that disrupts its interaction with adaptor molecules TIRAP and TRAM ${ }^{42}$ (Supplementary Fig. 5c). Similar to Tlr4-KO microglia, TAK-242-treated cells showed impaired p62 induction (Fig. 4d) as well as a reduction in the number of $h a-S y n /$ ubiquitin-positive puncta following ha-Syn treatment (Fig. 4e). Furthermore, Tlr4-KO mice injected with AAV-ha-Syn showed reduced p62 puncta number in Iba-1positive microglia compared with control mice (Fig. 4f, g), indicating that TLR4 is required for microglial p62 induction in response to ha-Syn exposure both in vivo and in vitro.

Lipopolysaccharide (LPS) triggers intracellular signaling via TLR4 binding, leading to the activation of the NF- $\kappa B$ and MAPK pathways. TLR4-LPS binding induces endocytosis of the TLR4LPS complex and subsequent expression of interferon-beta 1 (Ifnb1) through activation of interferon regulatory factor 3 (IRF3) ${ }^{43,44}$. Therefore, we asked if $h a-S y n$ triggers similar TLR4 
downstream events. Through RT-qPCR analysis, we found that p62 was also increased by LPS as reported ${ }^{45}$ (Fig. $4 \mathrm{~h}$ ); the major target genes of the NF- $\mathrm{KB}$ pathway, $I l-1 b$ and $T n f$, were upregulated by LPS or ha-Syn treatment. However, LPS has more robust effects in stimulating $I l-1 b$ and Tnf expression compared with ha-Syn (Fig. 4h). LPS, but not ha-Syn, induced Ifnb1 expression (Fig. 4h) and increased levels of p-IFR3 (S396), a marker of IRF3 activation (Fig. 4i), indicating the absence of IRF3 signaling in ha-Syn-treated microglia.

To test TLR4 endocytosis, we treated microglia with LPS or haSyn protein for $30 \mathrm{~min}$ and stained with TLR4 antibody conjugated with phycoerythrin (PE) to monitor the levels of TLR4 at the cell surface. LPS decreased the level of TLR4 on the plasma membrane as a result of endocytosis as reported ${ }^{44}$; by contrast, $h a-S y n$ had little effect on TLR4 levels at the cell surface (Fig. 4j), suggesting that ha-Syn does not induce TLR4 internalization. Lastly, pretreatment with Dynasore did not block p62 induction by ha-Syn (Fig. 4k). The above observations suggest that the ha-Syn-TLR4 interaction stimulates p62 expression without causing TLR4 receptor endocytosis distinctively from LPS-TLR4 signaling.

NF- $\mathrm{B}$ mediates $\alpha$-synuclein-TLR4 signaling and p62 induction. Upon ha-Syn treatment, microglia activate NF- $\mathrm{kB}$ and $\mathrm{p} 38$ pathways as indicated by an increase of $\mathrm{p}-\mathrm{NF}-\kappa \mathrm{B}$ (S536), a decrease of I $\kappa \mathrm{B}$ level, and an increase of p-p38 (T180/Y182) (Fig. 5a). However, p-ERK1/2 (T202/Y204) and p-JNK (T183/ Y185) were not changed (Fig. 5a). We next treated microglia with ML-120B, an inhibitor of IKK-2, which is responsible for I $\mathrm{B}$ phosphorylation and degradation that leads to NF- $\kappa B$ activation, and SB202190 or SB203580, two different inhibitors of p38. While the inhibitory effects of ML-120B, SB202190, and SB203580 were all confirmed in LPS-induced NF- $\kappa B$ activation and gene expression of proinflammatory cytokines (Supplementary Fig. 5d, e), only ML-120B significantly suppressed p62 induction and reduced the number of ha-Syn/ubiquitin-positive puncta after $h a-S y n$ treatment (Fig. $5 b, c$ ), suggesting that NF- $\kappa B$ is the primary mediator for TLR4-triggered p62 induction. Supporting this notion, mRNA levels of $I l-1 b$ and Tnf were significantly lower in Tlr4-KO than WT cells, despite that both WT and Tlr4-KO microglia show an increase in $I l-1 b$ and Tnf expression after ha-Syn treatment (Fig. 5d). By contrast, NRF2 or TFEB pathways were unaltered as shown by the lack of change in the level of NQO-1 and HO-1, two major targets of NRF2, or the intensity of nuclear TFEB in response to ha-Syn ${ }^{46,47}$ (Supplementary Fig. 4i, j).

TLR4 is a preferred TLR for $\alpha$-synuclein to activate NF- $\kappa B$. Previous studies suggest that in microglia $\alpha$-synuclein interacts with TLR2 (ref. ${ }^{19}$ ). We, therefore, tested whether the interaction between ha-Syn-TLR2 activates the NF- $\kappa$ B pathway. We applied a TLR2-blocking antibody (T2.5 clone), which suppressed p-NF$\kappa \mathrm{B}$ in response to the TLR2-specific ligand, $\mathrm{Pam}_{3} \mathrm{CSK}_{4}$ (Supplementary Fig. 5f). Remarkably, pretreatment with the TLR2blocking antibody completely suppressed the induction of $\mathrm{Il}-1 \mathrm{~b}$ and Tnf in response to ha-Syn in Tlr4-KO microglia (Fig. 5e). Interestingly, the TLR2-blocking antibody showed an enhanced effect of suppression in Tlr4-KO microglia compared with the WT (Fig. 5f), suggesting that TLR2 can partially compensate for TLR4 function in regulating the response to ha-Syn in Tlr4-KO microglia.

To test the specificity of ha-Syn effects on plasma membraneexpressed TLRs ${ }^{48}$, we established the NF- $\kappa \mathrm{B}$-luciferase system expressing NF- $\kappa B$ response element and luciferase reporter gene in HEK293T cells expressing TLR2, TLR2/TLR1, TLR2/TLR6,
TLR4/MD2/CD14 (TLR4 complex), or TLR5. First, we validated the specificity of this system by applying specific ligands for each TLR. Application of LPS, $\mathrm{Pam}_{3} \mathrm{CSK}_{4}$, and recombinant Flagellin peptides selectively increased NF- $\kappa \mathrm{B}$ activity in TLR4 complex, TLR2 and TLR2 combinations, and TLR5, respectively (Supplementary Fig. 6). Furthermore, ha-Syn treatment significantly increased NF- $\kappa \mathrm{B}$ activity in a dose-dependent manner in TLR4/ MD2/CD14-transfected cells compared with YFP transfected cells (Fig. 5g). We also observed that ha-Syn increased NF- $\kappa$ B activity in TLR2, TLR2/1, and TLR2/6-transfected cells comparable with the activity in the TLR4 complex, while no change of the NF- $\kappa B$ activity was observed in YFP or TLR5-transfected cells (Fig. 5h). Furthermore, we applied the TLR2-blocking antibody in cells cotransfected with TLR2 and the TLR4 complex. As expected, pretreatment with TLR2-blocking antibody prevented the increase of NF- $\kappa \mathrm{B}$ activity induced by $\mathrm{Pam}_{3} \mathrm{CSK}_{4}$, but not by LPS (Fig. 5i). Interestingly, application of the TLR2-blocking antibody had little effect on the increase of NF- $\mathrm{B}$ activity induced by ha-Syn (Fig. 5i). These results suggest that ha-Syn prefers TLR4 over TLR2 in regulating NF- $\mathrm{B}$ signaling, while haSyn may only act through TLR2 to induce NF- $\kappa B$ signaling in the absence of TLR4.

Autophagy-deficient microglia cause $\alpha$-synuclein accumulation. We next established microglia-specific Atg7-deficient mice (Cx3cr1 CreER-IRES-Eyfp; Atgflox/flox) by breeding Cx3cr1 CreER-IRES-Eyfp (tamoxifen-inducible Cre) with Atgfflox/flox mice. Although previous studies showed that deletion of Atg7 specifically in neurons causes neurodegeneration ${ }^{49,50}$, we found that Atg7-deficiency had little effect on microglia number and state-specific gene signatures (Supplementary Fig. 7a-g), indicating that loss of autophagy does not cause microglial death or activation for at least up to 7 months following Atg7 deletion.

We then tested the consequence of microglial Atg7-deficiency on a-synuclein homeostasis in the brain by investigating C $x 3$ crl CreER-IRES-Eyfp; Atg7flox/flox mice expressing ha-Syn through either AAV injection (Fig. 6a) or transgenic expression (Fig. 6c). After injection of AAV9-ha-Syn, we observed that Atg7deficient microglia contained more p62 puncta as well as p62/haSyn-positive puncta compared with control microglia (Supplementary Fig. 7h, i). Analysis of brain lysate fractions showed that microglial Atg7-deficient brains contained significantly elevated levels of detergent-insoluble ha-Syn at 6 weeks after AAV9-haSyn injection, as evidenced by a smear of high-molecular-weight ha-Syn species, compared to littermate controls (Fig. 6b).

We also examined microglial Atg7-deficient mice after crossing with $h a-S y n-T g(C \times 3 c r 1$ CreER-IRES-Eyfp; Atg7flox/flox; ha-Syn-Tg mice) at 7 months after Atg7 deletion (Fig. 6c). The number of p62 puncta and p62/ha-Syn-positive puncta was significantly higher in Atg7-deficient microglia than control (Fig. 6d, e). Furthermore, microglial Atg7-deficient brains contained elevated levels of detergent-insoluble $h a-S y n$ protein than control mice at 10 months of age (Fig. 6f). Furthermore, microglial Atg7-deficient mice showed increased staining of p-S129 a-synuclein-positive structures, particularly in the lateral SNpc (Fig. 6g, h) and the dorsal striatum (Fig. 6i, j).

Autophagy-deficient microglia promote neurodegeneration. Despite the lack of detectable neurodegeneration in ha-Syn-Tg

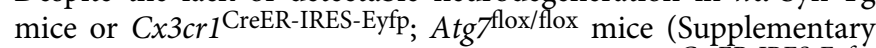
Fig. 1f; Fig. $7 \mathrm{c}, \mathrm{d})$, the compound mice $C \times 3 \mathrm{cr} 1^{\text {CreER-IRES-Eyfp; }}$ Atg $7_{\text {flox/flox; }}$ ha-Syn-Tg (after the cross of the above two lines of mice) exhibited a decrease in the number of dopaminergic neurons as shown by stereological counting TH-positive neurons and Nissl-positive cells in the SNpc (Fig. 7a, b). These data suggest 
a

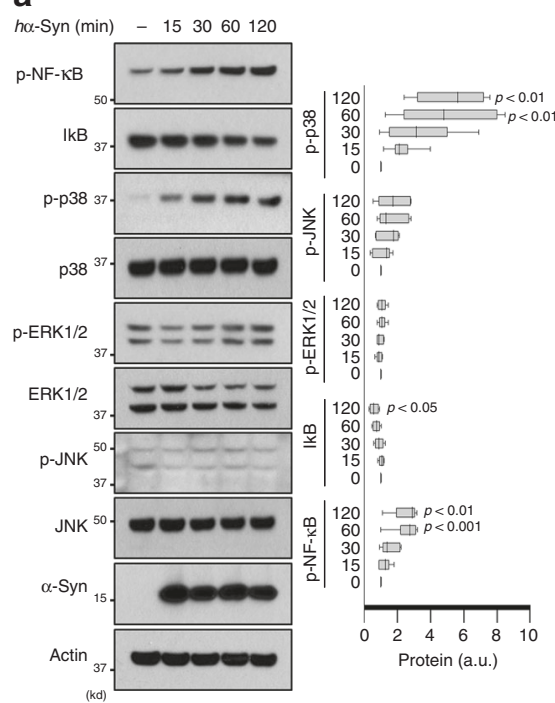

b ML120B _ _ $_{-} \quad \operatorname{SB} 202190(1 \mu \mathrm{M})-\quad+\quad+$

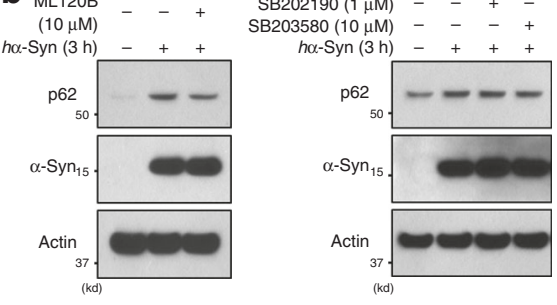

C

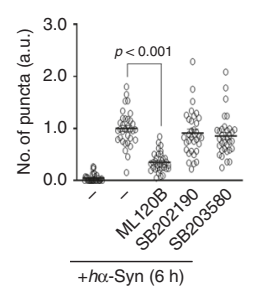

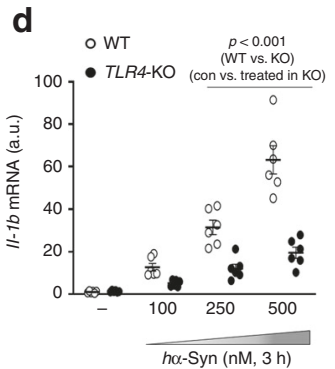
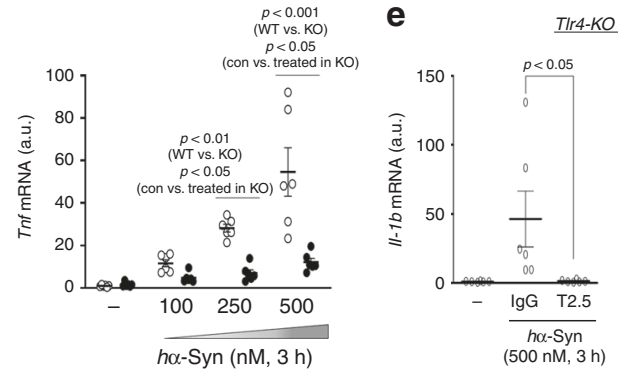

TIr4-KO microglia
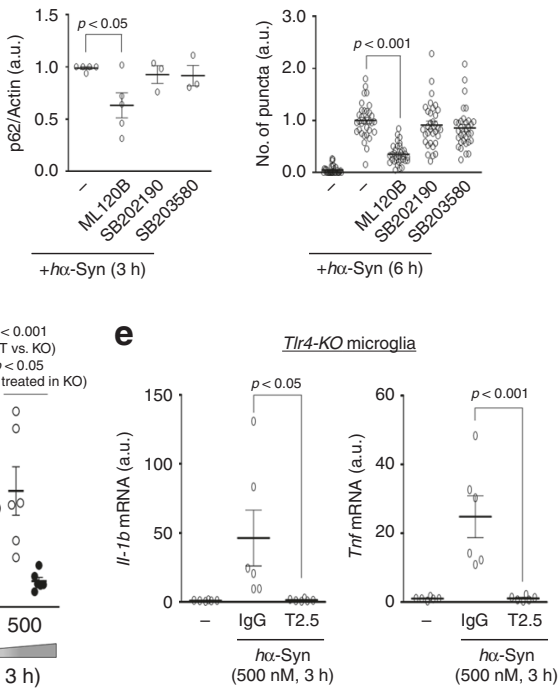

f

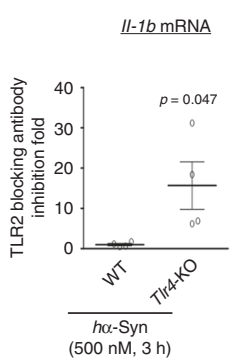

InfmRNA

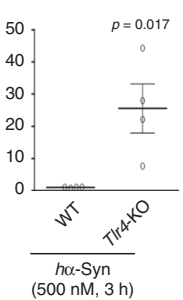

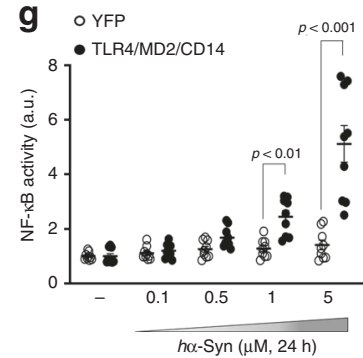

h

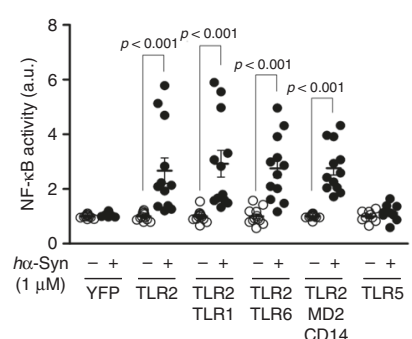

i $\circ \lg G$

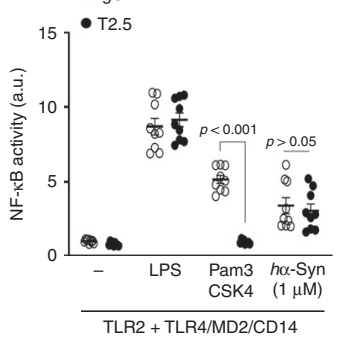

Fig. 5 NF-kB mediates $\boldsymbol{\alpha}$-synuclein-TLR4 signaling and p62 induction. a WT microglia were treated with $250 \mathrm{nM}$ h $\alpha$-Syn protein for the indicated time and assayed for W.B using antibodies as indicated. The levels of proteins were quantified in the right panel $(n=6)$. $p$-values were calculated by one-way ANOVA with Newman-Keuls post hoc test. $F(4,24)=9.196, p=0.0001$ for $p-N F-\kappa B, F(4,24)=4.530, p=0.0072$ for IkB, $F(4,24)=0.8644, p=0.4994$ for $p$-ERK1/2, $F(4,24)=1.369, p=0.2741$ for $p-J N K, F(4,24)=5.372, p=0.0031$ for $p-p 38$. b, c Cells were pretreated with ML-120B, an IKK-2 inhibitor, or SB203580 and SB202190, two different p38 inhibitors, for indicated concentrations before $250 \mathrm{nM}$ h $\alpha$-Syn protein treatment, and assayed for $W . B$ (b, $n=$ $3-5, F(3,12)=3.750, p=0.0413)$ or immunostaining $(\mathbf{c}, n=33, F(4,160)=56.21, p=1.53 E-29)$. The levels of proteins were quantified in the right panel. The number of $h \alpha$-Syn/ubiquitin-positive puncta was counted and quantified (c). p-values were calculated by one-way ANOVA with Newman-Keuls post hoc test. d-f Microglia obtained from WT mice and T/r4-KO mice were treated with $h \alpha-S y n$ protein for $3 \mathrm{~h}$ with indicated concentration without (d, $n=6$ ) or with $(\mathbf{e}(n=6), \mathbf{f}(n=4))$ TLR2-blocking antibody (T2.5), and assayed for RT-qPCR using primers for II-1b (left panel, F (3,40) $=20.70, p=2.95 \mathrm{E}-08$ for h $\alpha$-Syn-genotype interaction; $F(3,40)=69.43, p=6.55 \mathrm{E}-16$ for h $\alpha$-Syn; $F(1,40)=69.57, p=2.74 \mathrm{E}-10$ for genotype) and Tnf (right panel, $\mathrm{F}(3,40)=10.12$, $p=4.32 \mathrm{E}-05$ for h $\alpha$-Syn-genotype interaction; $\mathrm{F}(3,40)=21.73, p=1.64 \mathrm{E}-08$ for h $\alpha$-Syn; $\mathrm{F}(1,40)=34.36, p=7.39 \mathrm{E}-07$ for genotype). $p$-values were calculated by two-way ANOVA with Bonferroni post hoc test (wt vs ko in d), one-way ANOVA with Newman-Keuls post hoc test (non-treated vs. treated in d, e; $F(3,20)=22.11, p=1.46 \mathrm{E}-06$ for $\|-1 b, F(3,20)=11.88, p=0.0001$ for Tnf), and unpaired two-tailed Student's $t$ test (f). g-i HEK293T cells were transfected with a luciferase vector that expresses luciferase under the promoter containing NF-kB-binding elements, and various TLRs. Then, cells were treated $h \alpha$-Syn protein with indicated concentration $(\mathbf{g}(n=9), \mathbf{h}(n=9-12), \mathbf{i}(n=9)), 50 \mathrm{ng} / \mathrm{ml}$ LPS $(\mathbf{i})$, or Pam $\mathrm{CSK}_{4}(\mathbf{i})$ for $24 \mathrm{~h}$ without $(\mathbf{g}, \mathbf{h})$ or with (i) $10 \mu \mathrm{g} / \mathrm{ml}$ TLR2-blocking antibody (T2.5) and control lgG. $p$-values were calculated by two-way ANOVA with Bonferroni post hoc test. In $\mathbf{g}$, F(4,80) $=$ 19.63, $p=2.74 \mathrm{E}-11$ for interaction; $\mathrm{F}(4,80)=27.31, p=2.66 \mathrm{E}-14$ for h $\alpha-\mathrm{Syn} ; \mathrm{F}(1,80)=47.95, p=9.90 \mathrm{E}-10$ for protein overexpression. In $\mathbf{h}, \mathrm{F}(5,126)=$ $6.070, p=4.54 \mathrm{E}-05$ for interaction; $\mathrm{F}(5,126)=6.070, p=4.54 \mathrm{E}-05$ for h $\alpha-S y n ; F(1,126)=73.83, p=2.77 \mathrm{E}-14$ for protein overexpression. In $(\mathbf{i}), \mathrm{F}(3,64)$ $=16.98, p=3.19 \mathrm{E}-08$ for interaction; $\mathrm{F}(3,64)=177.4, p=5.90 \mathrm{E}-31$ for stimuli; $\mathrm{F}(1,64)=17.47, p=9.03 \mathrm{E}-05$ for antibody. Data are representative of at least three independent experiments. All values are reported as mean \pm SEM.

that disruption of microglial autophagy significantly enhances $\alpha$ synuclein-mediated neurotoxicity. Taken together, our results demonstrate an important role for microglial autophagy in clearing a-synuclein released from neurons and preventing neurodegeneration.

\section{Discussion}

Our study has uncovered a significant role of microglia in clearing extracellular a-synuclein and protecting neurons through selective autophagy, which we term "synucleinphagy". Here, we show the first evidence that microglia sequester engulfed $a$-synuclein into autophagosomes for lysosomal degradation, thus solving a long-standing question as to whether wild-type a-synuclein is indeed degraded by autophagy. We identified the mechanism for microglia-specific synucleinphagy, which is mediated through TLR4-NF- $\mathrm{KB}$ signaling by transcriptional upregulation of the autophagy receptor, p62/SQSTM1 (Fig. 7e). By contrast, neuron has no change of autophagosomes or p62 protein levels after the treatment with various forms of $\alpha$-synuclein ${ }^{51}$, likely due to lack of the TLR4-NF- $\kappa \mathrm{B}$ pathway.

We demonstrate the critical role of TLR4-NF- $\mathrm{BB}-\mathrm{p} 62$ in mediating synucleinphagy in microglia. TLR4 signaling has been linked to xenophagy, a selective autophagic degradation of 
a

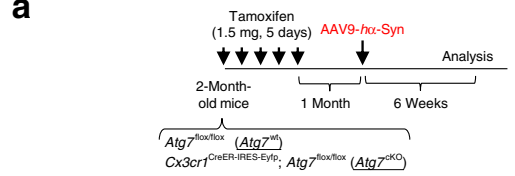

b

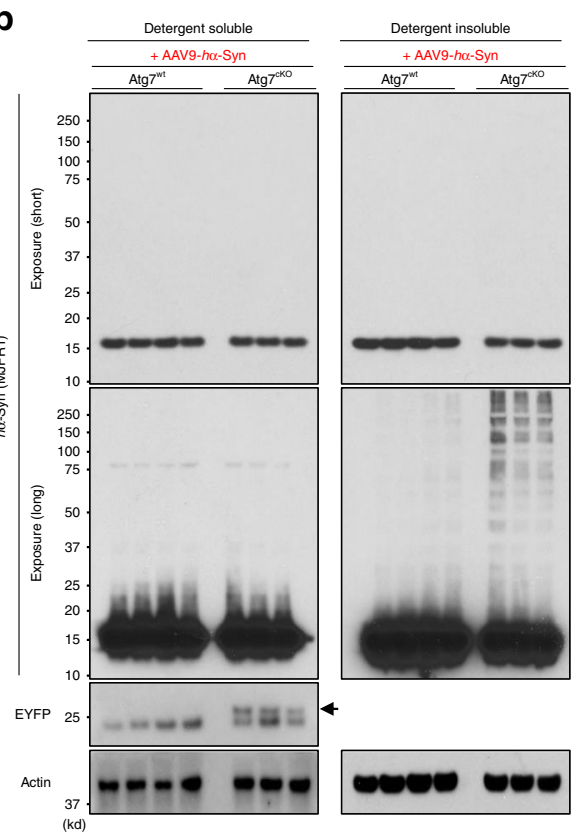

C

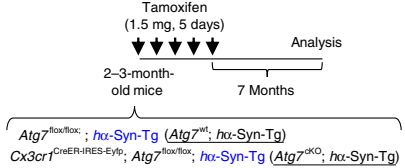

d

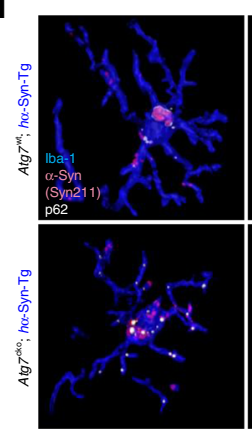

e

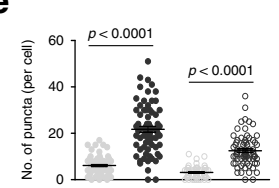

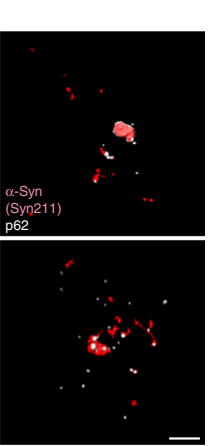

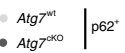

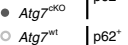

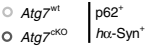

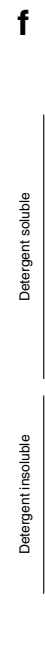

g

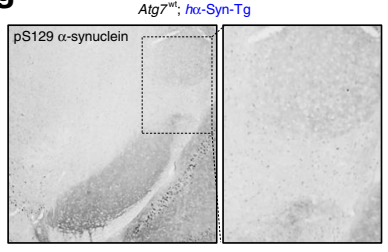

i

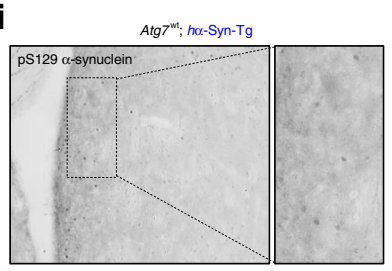

f
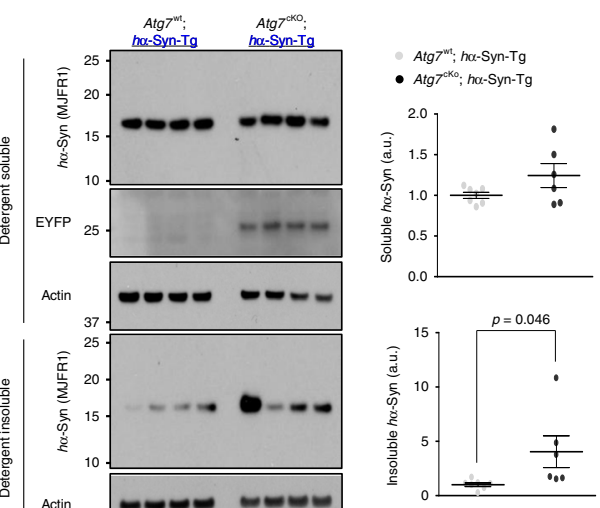

Fig. 6 Autophagy-deficient microglia cause $\boldsymbol{\alpha}$-synuclein accumulation. a Experimental plan to test the roles of microglia-specific Atg7-deficiency in AAV-

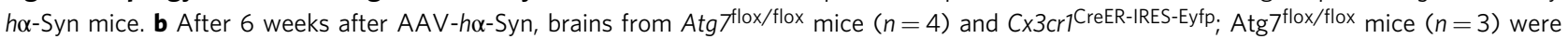
homogenized and fractionated into a detergent-soluble fraction and detergent-insoluble fraction and assayed for W.B using antibodies against human $\alpha$ synuclein, and GFP/YFP. Actin were used as loading controls. Arrow indicates the EYFP band. c Experimental plan to test the roles of microglia-specific Atg7-deficiency in $h \alpha$-Syn-Tg mice. d, e At 7 months after tamoxifen treatment, brain slices were fixed and stained with antibodies against human $\alpha$ synuclein, p62, and Iba-1. Representative 3D reconstruction pictures of microglia containing p62 and human $\alpha$-synuclein in the striatum of Atg flox/flox mice

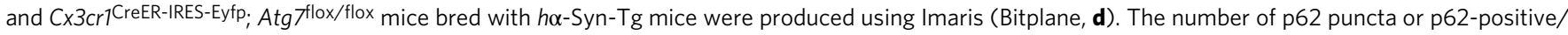

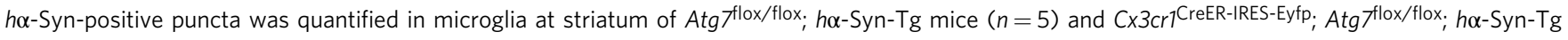
mice $(n=5)(\mathbf{e})$. $p$-values were calculated by two-tailed Mann-Whitney $U$ test. Scale bar, $10 \mu \mathrm{m}$. $\mathbf{f}$ At 7 months after tamoxifen treatment, brains from Atg flox/flox; $h \alpha$-Syn-Tg mice $(n=7)$ and Cx3cr7CreER-IRES-Eyfp; Atg fflox/flox; $h \alpha-S y n-T g$ mice $(n=6)$ were homogenized and fractionated into detergentsoluble fraction and detergent-insoluble fraction, and assayed for W.B using antibodies against human $\alpha$-synuclein, and GFP/YFP for confirming the presence of $\mathrm{Cx}_{3} \mathrm{Cr}_{7} \mathrm{CreER-IRES-Eyfp}$. Band intensities were quantified in the lower panel. $p$-values were calculated by unpaired two-tailed Student's $t$ test. $\mathbf{g}$-j $\mathbf{p}$ S129- $\alpha$-synuclein-positive structures were visualized in brain sections from Atg flox/flox; $\alpha$-Syn-Tg mice $(n=7)$ and Cx3cr7CreER-IRES-Eyfp; Atgflox/flox; $h \alpha-$ Syn-Tg mice $(n=6)$ through DAB staining method $(\mathbf{g}, \mathbf{i})$, and quantified $(\mathbf{h}, \mathbf{j}) . p$-values were calculated by unpaired two-tailed Student's $t$ test. Scale bar, $100 \mu \mathrm{m}$. All values are reported as mean \pm SEM. Data are representative of at least three independent experiments.

infected bacteria, in macrophages. TLR4-NF- $\kappa \mathrm{B}$ signaling was previously shown to induce selective autophagy after pathogen invasion $^{52-54}$. In addition, p62 has a role in the clearance of intracytosolic Shigella and Listeria ${ }^{55}$. Thus, synucleinphagy and xenophagy have a shared mechanism through TLR4-mediated signaling. Our study, however, showed that synucleinphagy require specifically p62 as other autophagy receptors, such as Ndp52, Optineurin, or Nbr1, are not upregulated upon TLR4$\mathrm{NF}-\kappa \mathrm{B}$ activation. We also noted that during synucleinphagy, LC3-II level was unaltered, despite the increase of p62 levels and colocalization of LC3 puncta with ingested a-synuclein in microglia, suggesting a lack of global upregulation of autophagy but rather a restricted, local selective autophagy. The specific sequestration of $\alpha$-synuclein into autophagosomes is likely mediated by p62 binding of ubiquitin-linked $\alpha$-synuclein protein $^{34}$; future study should identify the specific E3 ligase of $\alpha$ synuclein in microglia responsible for synucleinphagy. Moreover, synucleinphagy differs from aggrephagy, a selective type of autophagy which degrades intracellular protein aggregates without the involvement of cell surface receptors (e.g., TLR) or NF- $\kappa B$ signaling.

Our result reveals that $\alpha$-synuclein has a strong preference for TLR4 in signaling NF- $\kappa$ B activity over TLR2 receptor, despite that $\alpha$-synuclein can act through either receptor, in an agreement with previous reports showing that both receptors interact with $\alpha$ synuclein (albeit different forms). For example, TLR4 was required for NF- $\mathrm{kB}$ activation, and increased inflammation and phagocytic activity in response to various forms of $\alpha$ synuclein $40,41,56$. By contrast, TLR2 also plays a role in neurons in response to a-synuclein in addition to microglia $19,57,58$. Future 

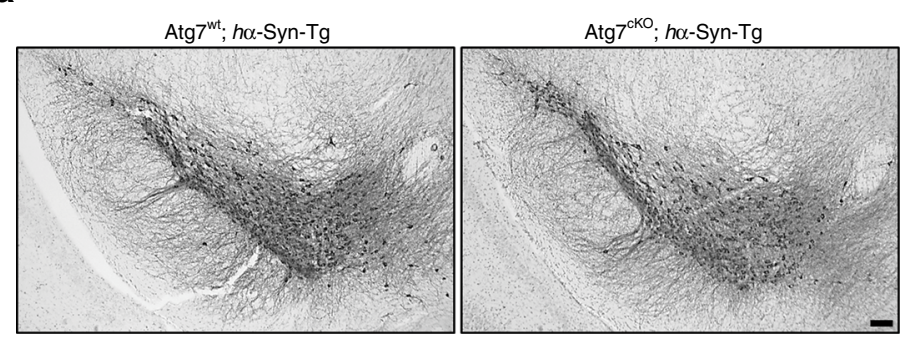

C
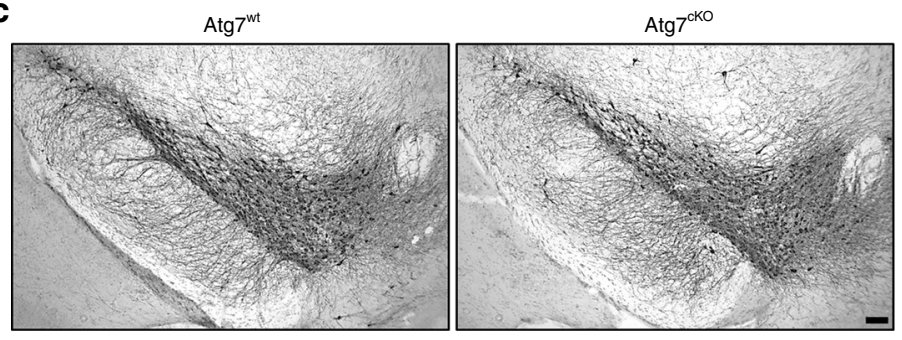

b
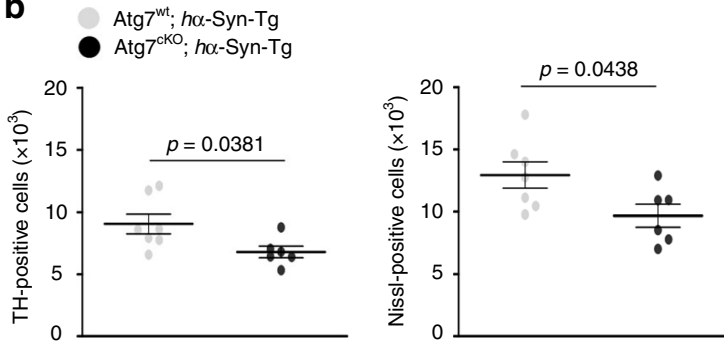

d

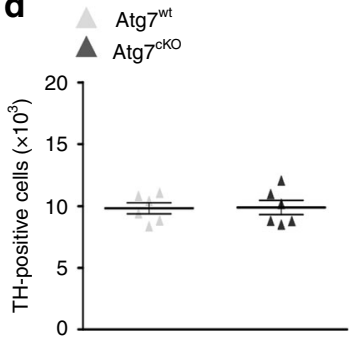

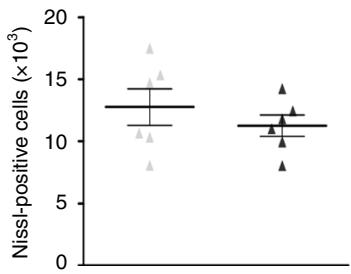

e

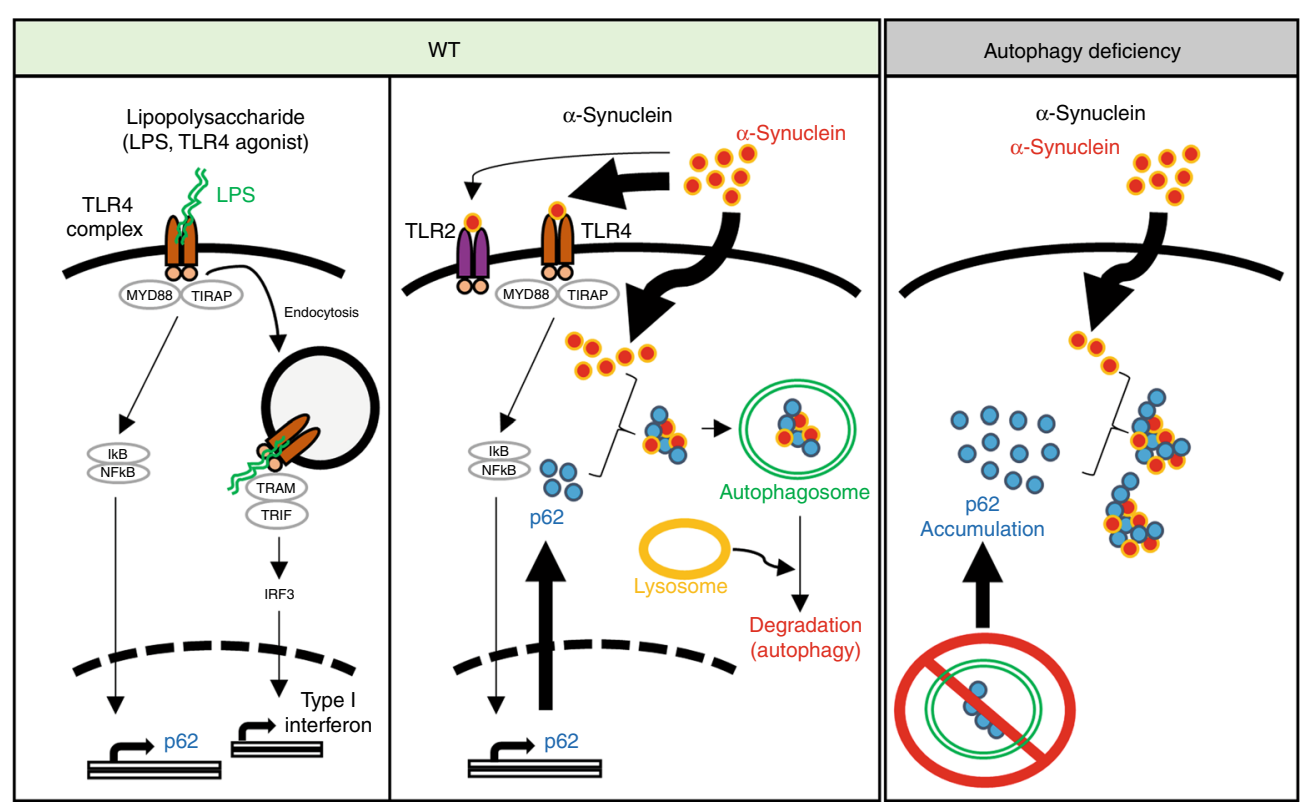

Fig. 7 Autophagy-deficient microglia promote neurodegeneration. a, b At 7 months after tamoxifen treatment, brains from Atg 7 flox/flox; $h \alpha-S y n-T g$ mice $(n=7)$ and $C \times 3 \mathrm{cr}$ CreER-IRES-Eyfp; Atg flox/flox; $h \alpha$-Syn-Tg mice $(n=6)$ were fixed and stained using an antibody against tyrosine hydroxylase (TH) using DAB method (a). TH-positive cells and Nissl-positive cells in the SNpc area were counted (b). p-values were calculated by unpaired two-tailed Student's $t$ test. Scale bar, $100 \mu \mathrm{m}$. c, d At 12 months after tamoxifen treatment, TH-positive cells and Nissl-positive cells were counted in SNpc area of Atgflox/flox $(n=6)$ and $C \times 3 c r 7^{C r e E R-I R E S-E y f p ;}$ Atg flox/flox $_{(n=6)}$ brains. e Hypothetical model of microglial synucleinphagy. All values are reported as mean \pm SEM.

studies should investigate further the significance of other TLRs in mediating $\alpha$-synuclein degradation as well as inflammation in microglia. Our study also reveals a distinct feature of $\alpha$-synucleintriggered TLR4 activation, which does not promote TLR4 endocytosis in contrast to the effect of LPS. The absence of endocytosed TLR4 may prevent Ifnb1 induction or increase of p-IFR3 (S396) level, despite the activation of NF- $\kappa \mathrm{B}$ signaling, thus avoiding excessive inflammation and neurotoxicity ${ }^{59}$.

Our report, however, raises many questions with regard to the exact mechanism whereby a-synuclein enters microglia. Our study suggests that microglia ingest and sequester $\alpha$-synuclein into autophagosomes independent of endocytosis or phagocytosis. Thus, synucleinphagy is a distinct process from LC3associated phagocytosis (LAP) ${ }^{60}$. A number of studies demonstrated that $a$-synuclein can enter the cells or penetrate cytoplasmic membrane through endocytosis-independent process $^{61-63}$. Therefore, our study supports a model that a portion of extracellular $\alpha$-synuclein interacts with TLR4 (or TLR2), which then triggers NF- $\mathrm{kB}$ signaling, while a majority of $\alpha$ synuclein penetrates cytoplasmic membrane or enters microglia through TLR4 endocytosis-independent manner. The precise process and mechanism for $\alpha$-synuclein to enter microglia awaits future detailed investigation.

Finally, emerging human genomic studies suggest the autophagy-lysosome system as a converging pathogenic pathway of $\mathrm{PD}^{64}$, and many PD-related genes such as PINK1, PARKIN, GBA, and $L R R K 2$, were shown to play a role in autophagy-related pathways in glial cells ${ }^{65}$. Our finding of microglial synucleinphagy via selective autophagy that confers neuroprotection may assist in understanding the role of microglia in preventing synucleinopathies. 


\section{Methods}

Animals. B6.B10ScN-Tlr4lps-del/JthJ mice (Tlr4-KO, \#007227), B6J.B6N(Cg)

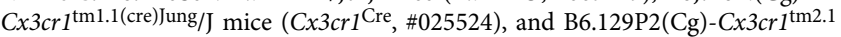
(cre/ERT2)Litt/WganJ mice (Cx3cr1 CreER-IRES-Eyfp in this study, \#021160) were purchased from Jackson Laboratory (Bar Harbor, ME). Atg $7^{\text {flox/flox }}$ mice and $p 62-\mathrm{KO}$ mice were kindly gifted from Dr. Masaaki Komatsu (Tokyo, Japan) ${ }^{38,66}$. Atg14 1 flox/ flox mice were Dr. Herbert W.Virgin (Washington University School of Medicine, St. Louis, MO) ${ }^{39,67}$. GFP-LC3 transgenic mice (C57BL/6J) was described previously $^{35}$. For generating microglia-specific Atg7-deficient mice, Atg 7 flox/flox mice

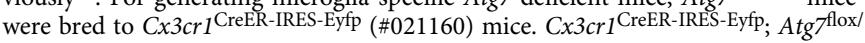

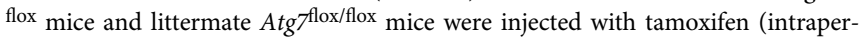
itoneal injection, 5 continuous days, $1.5 \mathrm{mg}$ per mouse) to induce Cre recombinase

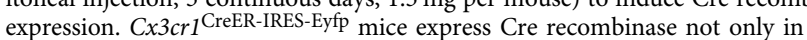
microglia but also in myeloid cell populations in the blood which are replenished by $\mathrm{Cx} 3 \mathrm{cr} 1$-negative progenitors, which takes 1 month. However, due to limited turn-over, microglia will continuously express Cre recombinase ${ }^{33}$. Therefore, we injected AAV-ha-Syn after 1 month post tamoxifen administration to avoid any possible involvement of Atg7-deficient blood-derived cells in our observation. For obtaining Atg7 or Atg14-deficient cultured cells, Atg $7^{\text {flox/flox }}$ mice or Atg14 $4^{\text {flox/flox }}$ mice were bred to $C \times 3 c r 1^{\text {Cre }}$ mice (\#025524). Mice were maintained under a 12 -h light/12-h dark cyclerooms with water accessible at all times. All animal procedures were approved by Icahn School of Medicine at Mount Sinai Animal Care and Use Committee (IACUC-2015-0046).

\section{Generation of Thy-1-SNCA mice (Jax \#017682). The transgenic construct} contained a $4.2 \mathrm{~kb}$ murine Thy-1 (Gene location on chromosome 9: 43,851,467$43,856,662$ ) promoter fragment containing $\sim 1.6 \mathrm{~kb}$ of $5^{\prime}$ flanking sequence plus the $5^{\prime}$ first UTR exon $(93 \mathrm{bp})$, the first intron $(2480 \mathrm{bp})$, and the noncoding region of exon 2 before ATG. This was followed by a 423 bp ORF of human wild-type $\alpha-$ synuclein cDNA (GenBank Acc\# NM_000345), and 221 bp of SV40 polyA sequence in a pGL3 vector backbone (Promega). The above strategy was according to the published paper ${ }^{68}$. The microinjection fragment was purified and quantified before the injections. Three injection sessions were performed into C57BL/6Ntac embryos. Founders were identified and mated to wild-type C57BL/6NTac females. Analysis of integration site indicates insertion occurred at Chr11:4045678740495044 and resulted in a $38.4 \mathrm{~Kb}$ deletion ${ }^{69}$. Copy number analysis was also performed on tail clips of the mice using the following primers and probe combination (spanning exons 2 and 3 of hSNCA gene):

Forward primer: $5^{\prime}$-AGGACTTTCAAAGGCCAAGG- $3^{\prime}$

Probe (FAM-labeled): 5'-AGTTGTGGCTGCTGCTGAGAAAACC-3'

Reverse primer: 5'-CACTTGCTCTTTGGTCTTCTCAG-3'

The DNA copy number was determined by a standard curve method where for the standard curve the calculated number of copies of $(1,5,10,20,50$, and 100 copies) of the $5.1 \mathrm{~kb}$ transgene DNA fragment was mixed with $5 \mathrm{ng}$ of DNA prepared from the wild-type $\mathrm{C} 57 \mathrm{BL} / 6 \mathrm{NTac}$ mice. The data were analyzed by plotting $\Delta \mathrm{Ct}$ values against the known copy number. A standard curve was run in parallel with the samples prepared from the mice. An average copy number was $1.98+/-0.81$. Following copy number analysis, the line was sent to The Jackson Laboratory for colony establishment (C57BL/6N-Tg (Thy-1-SNCA)15Mjff/J; Stock No. 017682).

Adeno-associated virus (AAV). AAV vectors encoding either GFP or human WT a-synuclein were kindly gifted from Dr. Jia-Yi Li (Lund University, Lund Sweden). AAV9-GFP or AAV9-human WT a-synuclein were packaged using the service provided by Vigene company (Rockville, MD). For establishing an acute PD animal model, $2 \mu \mathrm{l}$ of $6 \times 10^{13} \mathrm{GC} / \mathrm{ml}$ of either AAV-GFP or AAV- $\alpha$-synuclein were injected into the SNpc area of mice (AP: $-3.28 \mathrm{~mm}, \mathrm{ML}:-1.5 \mathrm{~mm}, \mathrm{DV}:-4.1$ $\mathrm{mm}$ ) using a stereotaxic apparatus (Kopf Instruments, Tujunga, CA).

Cell culture. Primary microglia were obtained from mixed glia cultured from forebrain of pups from 1- to 3-day-old C57/BL6J WT mice (Jackson Laboratory). Briefly, forebrains were isolated and homogenized into single-cell suspensions by triturating with fire-polished Pasteur pipettes in Dulbecco's modified Eagle's medium (DMEM, \#11965-092, Gibco ${ }^{\text {TM }}$, Thermo Fisher Scientific) containing penicillin/streptomycin (\#15080-063, Gibco $\left.{ }^{\mathrm{TM}}\right)$, GlutaMAX ${ }^{\mathrm{TM}}$ (\#35050061, Gib$\mathrm{co}^{\mathrm{TM}}$ ), and heat-inactivated $10 \%$ fetal bovine serum (FBS, \#S11550, Atlanta Biologicals, Atlanta, GA). Homogenized tissues were plated at $75 \mathrm{~cm}^{2}$ T-flask (BD Bioscience, San Jose, CA, USA) and incubated for 2 weeks. Floating microglia were detached from flasks by mild shaking and then filtered through a $70-\mu \mathrm{m}$ cell strainer to remove cell clumps or debris. Microglia were plated onto culture dishes at an appropriate density. HEK293T cell line was maintained in DMEM supplemented with penicillin/streptomycin and 10\% FBS.

Adult microglia isolation. For adult microglia isolation, we adopted the established protocol ${ }^{32,70}$. Briefly, whole brains were isolated from mice after whole-body intracardial perfusion with ice-cold D-PBS (Gibco ${ }^{\mathrm{TM}}$ ) and homogenized using $15-\mathrm{ml}$ size Dounce homogenizer in $5 \mathrm{ml}$ ice-cold D-PBS with ten strokes. Homogenized tissues were filtered through a $70-\mu \mathrm{m}$ cell strainer to remove any debris and centrifuged with $1000 \times g$ for $10 \mathrm{~min}$ at $4{ }^{\circ} \mathrm{C}$. Cell pellets were resuspended in $5 \mathrm{ml}$ of $70 \%$ Percoll $^{\oplus}$ (P1644, Sigma-Aldrich) made in $10 \%$ FBScontaining DMEM and $7 \mathrm{ml}$ of $40 \%$ Percoll $^{\top}$ made in D-PBS were laid on the top of it gently in a $15 \mathrm{ml}$ tube $\left(\right.$ Falcon ${ }^{\mathrm{TM}}$, CA). After centrifugation with $800 \times \mathrm{g}$ speed for $30 \mathrm{~min}$ in RT, top layer enriched with myelin was removed and the middle layer $(\sim 10 \mathrm{ml})$ was mixed with ice-cold D-PBS $(\sim 40 \mathrm{ml})$, and centrifuged with $1400 \times g$ for $5 \mathrm{~min}$ at $4{ }^{\circ} \mathrm{C}$. This microglia-enriched collection was further used for flow cytometry.

Flow cytometry. Microglia-enriched pellets were resuspended in $500 \mu \mathrm{l}$ of FACS buffer (1\% FBS in ice-cold D-PBS), and incubated with $10 \mu \mathrm{g} / \mathrm{ml}$ of anti-mouse CD16/CD32 (\#14-0161-82, Invitrogen) for $15 \mathrm{~min}$ on the ice to block Fc-receptor on microglia followed by $5 \mu \mathrm{g} / \mathrm{ml}$ of CD45 monoclonal antibody (I3/2.3) conjugated with APC-Cyanine7 (\#A15395, Invitrogen) and $2 \mu \mathrm{g} / \mathrm{ml}$ of CD11b monoclonal antibody (M1/70) conjugated with PerCP-Cyanine5.5 (\#45-0112-82, Invitrogen) for $30 \mathrm{~min}$ on the ice. After washed, the microglia population $\left(\mathrm{CD} 11 \mathrm{~b}^{\text {High }} / \mathrm{CD} 45^{\text {Intermediate }}\right)$ was gated and collected as shown in Supplementary Fig. 2 using FACSDiva v8.0.1 (BD Biosciences). In an initial validation step, microglia from $\mathrm{C} \times 3 \mathrm{cr} 1^{\text {CreER-IRES-Eyfp }}$ were stained with CD45 and CD11b antibodies and the ratio of $\mathrm{CD} 11 \mathrm{~b}^{\mathrm{High}} / \mathrm{CD} 45^{\text {Intermediate }}$ cell population among EYFPpositive cells, and vice versa was checked by setting each other's cell population as a parent gate as shown in Supplementary Fig. 2.

Quantitative RT-PCR. cDNA was synthesized from the total RNA with AffinityScript ${ }^{\mathrm{m}}$ Multi-Temp RT (Stratagene) with oligo dT18 as primer. For real-time PCR, PlatinumTaq DNA polymerase (Invitrogen) and a SYBR green (Molecular Probes) containing buffer were used. The real-time PCRs were performed using a thermocycler (ABI7900HT; Applied Biosystems). The PCR conditions used were: $95^{\circ} \mathrm{C}$ for $2 \mathrm{~min}, 40 \mathrm{cycles}$ of $95^{\circ} \mathrm{C}$ for $15 \mathrm{~s}, 55^{\circ} \mathrm{C}$ for $15 \mathrm{~s}$, and $72^{\circ} \mathrm{C}$ for $30 \mathrm{~s}$ using the following primer pairs (Eurofins MWG Operon):

Actin, 5' -AGGTGACAGCATTGCTTCTC-3' (sense), 5'

GCTGCCTCAACACCTCAAC-3' (antisense);

Axl, 5'-AGCACAGTCTGCAAACTCC-3' (sense), 5'-

CTACCTCTAGCTCCGTAGGTT-3' (antisense);

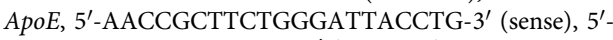

CTCTCCCTCGGCTAGGCAT-3' (antisense);

Cd9, 5'-TAACTTCATCTTCTGGCTCGCT-3' (sense), $5^{\prime}$ -

AAACCAACCAGCATCATGAGG-3' (antisense);

Clec $7 a, 5^{\prime}$-ACTTCAGCACTCAAGACATCC-3' (sense), 5'-

TGGCTTCCTTTCTCTGATCC-3' (antisense);

$C s f 1 r, 5^{\prime}$-CCTCAAACGTGGAGACACCAA- $3^{\prime}$ (sense), $5^{\prime}$ -

CGTGTGCCAACATCATTGCT-3' (antisense);

Cst $7,5^{\prime}$-TTCAACAACTGCACAAATGACA-3' (sense), 5' -

GGCCTTTCACCACCTGTACCA-3' (antisense);

C $x 3 c r 1,5^{\prime}$-CAACCCCTTTATCTACGCCTT-3' (sense), 5'-

GACCCATCTCCCTCGCTTG-3' (antisense);

HexB, 5'-TACAAGAACCAGTAGCCGTCCT-3' (sense), 5'

CTCTAAACCTCGTAACGCTCC-3' (antisense);

$H 2-A b 1,5^{\prime}$-GCCCTCAACCACCACAACAC-3' (sense), 5'-

AGTCCCCATTCCTAATAAGCTGT-3' (antisense);

$I l-1 b, 5^{\prime}$-CCTCTGATGGGCAACCACTT-3' (sense), $5^{\prime}$ -

TTCATCCCCCACACGTTGAC-3' (antisense);

Il-6, 5' -ACAGAAGGAGTGGCTAAGGA-3' (sense), $5^{\prime}$ -

CGCACTAGGTTTGCCGAGTA- $3^{\prime}$ (antisense);

Ifnb1, 5'-AGATGTCCTCAACTGCTCTC-3' (sense), 5'

AGATTCACTACCAGTCCCAG-3' (antisense);

Ifng, 5'-TGGCAGGAGATGTCTACACT-3' (sense), 5' -

GAAGCACCAGGTGTCAAGTC-3' (antisense);

Itgax, $5^{\prime}$-CTGCTGCCACCAACCCTTC-3' (sense), $5^{\prime}$

AGCCATCAATCAGGAACACGA-3' (antisense);

$L p l, 5^{\prime}$-ACAAGTTTTAGAGCAGGACCAT-3' (sense), 5' -

TTGCACAGCAGTTTACAAGCATC- $3^{\prime}$ (antisense);

Olfml3, 5' - GACACAGAACCCAGCTTTGC-3' (sense), 5' -

GCTACAGTCCGTCACCATATCGT-3' (antisense);

p62/Sqstm1, 5'-GAAGCTGCCCTATACCCACA-3' (sense), 5'

CCCGATGTCGTAATTCTTGGTC-3' (antisense);

P2ry 12, 5'-TTTGCTGGGCTCATCACGAAC-3' (sense), $5^{\prime}$ -

ACTGAAGTAACTTGGCACACC-3' (antisense)

Tgfbr 1, 5'-GATCCATCACTAGATCGCCCTT-3' (sense), 5'

CCGACCTTTGCCAATGCTT-3' (antisense);

Tmem 119, 5'-CTGACATTCTGGCTGCTACC-3' (sense), 5'

CACCCTTCACAGGCTTTGCTC-3' (antisense);

Tnf, $5^{\prime}$-TCACTGGAGCCTCGAATGTC-3' ${ }^{\prime}$ (sense), $5^{\prime}$

GTGAGGAAGGCTGTGCATTG-3' (antisense);

Trem $2,5^{\prime}$-GTCCCAAGCCCTCAACACC-3' (sense), $5^{\prime}$

TCCTCACCCAGCTGCCGACA-3' (antisense)

The cycle threshold $(\mathrm{Ct})$ for the gene transcript was normalized to the average Ct for transcripts of the housekeeping gene, Actin, amplified in each reaction. Relative quantitation of normalized transcript abundance was determined using the comparative Ct method $(\Delta \Delta \mathrm{Ct})$. 
Immunostaining. For brain tissue staining, mice were anesthetized and transcardially perfused with ice-cold PBS, and brains were obtained. Then, brains were fixed overnight at $4{ }^{\circ} \mathrm{C}$ in $4 \%$ paraformaldehyde. Fixed brains were stored at $4{ }^{\circ} \mathrm{C}$ in a $30 \%$ sucrose solution until they sank. A series of coronal sections $(30 \mu \mathrm{m})$ were obtained with a cryostat (Leica, Wetzlar, Germany). Coronal sections were incubated with blocking/permeabilization buffer ( $5 \%$ goat serum and $0.25 \%$ Triton $\mathrm{X}-100$ in PBS) at RT for $30 \mathrm{~min}$ and incubated with primary antibodies against Aquaporin 4 (AB3594, Millipore, 1:500), GFAP (\#130300, Thermofisher Scientific, 1:500), GFP/YFP (MA5-15256, Thermofisher Scientific, 1:500), Iba-1 (019-19741, Wako, Japan, 1:500), NeuN (MAB377, Chemicon, 1:500), p62 (GP62-C, Progen, Germany, 1:250), a-synuclein (clone MJFR1, ab138501, Abcam, 1:500; clone syn211 conjugated with Alexa594 fluorescein, sc-12767 AF594, Santa Cruz Biotechnology, 1:200), or ubiquitin (clone P4D1, sc-8017, Santa Cruz Biotechnology, $1: 500)$ at $4{ }^{\circ} \mathrm{C}$ overnight followed by secondary antibodies conjugated with Alexafluorescein. For 3,3'-diaminobenzidine (DAB) staining, serial sections were rinsed three times with $\mathrm{PBS}$, treated with $3 \% \mathrm{H}_{2} \mathrm{O}_{2}$ for $3 \mathrm{~min}$, and rinsed with $\mathrm{PBS}$ containing $0.1 \%$ Triton X-100 (PBST). After incubating in blocking solution (10\% Goat serum and $0.25 \%$ Triton X-100 in PBS), sections were incubated overnight at room temperature with primary antibodies against Tyrosine hydroxylase (AB152, Millipore, 1:1000) and p-S129 $\alpha$-synuclein (ab51253, Abcam, 1:1000). After rinsing in PBST, sections were incubated with biotinylated secondary antibodies (Vector Laboratories, Burlingame, CA, USA) for $1 \mathrm{~h}$ and the avidin/biotin system (Vector Laboratories) for $30 \mathrm{~min}$ and visualized using a DAB solution (Vector Laboratories). Sections were then mounted on gelatin-coated slides and examined under a bright-field microscope (Olympus Optical, BX51, Tokyo, Japan). For cultured primary microglia, cells were fixed with $4 \%$ paraformaldehyde at $4{ }^{\circ} \mathrm{C}$ for $15 \mathrm{~min}$ and incubated with microwave-boiled antigen retrieval solution (\#CTS013, R\&D System) for $5 \mathrm{~min}$. After washing with PBS, cells were incubated with blocking/ permeabilization buffer (1\% BSA and $0.1 \%$ Triton X-100 in PBS) at RT for $30 \mathrm{~min}$ and incubated with primary antibodies including EEA1 (610457, BD Bioscience, 1:100), $\alpha$-synuclein (clone MJFR1, ab138501, Abcam, 1:500; clone 2f12,

MABN1817, Millipore; clone 42, 610787 BD Bioscience, 1:100; clone 204, \#2647, Cell Signaling, 1:100), p62 (GP62-C, Progen, Germany, 1:250), ubiquitin (clone P4D1, sc-8017, Santa Cruz Biotechnology, 1:100), and TFEB (A303-673A, Bethyl Laboratories Inc, TX, 1:200) at $4{ }^{\circ} \mathrm{C}$ overnight followed by secondary antibodies. Images were obtained using a confocal microscope with Zen 2011 software (Zeiss LSM 780, Carl Zeiss, Jena, Germany).

\section{Microglia cell number and morphology analysis. From two brain slices from} each animal, z-stack pictures were obtained with $20 \times$ magnification at multiple areas of the striatum using a confocal microscope after staining with Iba-1 antibody (Zeiss LSM 780, Carl Zeiss, Jena, Germany). Then, microglial cell number was counted from z-stack projected pictures using "cell counter" plug-in in Fiji software (National Institutes of Health, MD). For morphology analysis, the length of processes and terminal points of branches were measured by a semi-automated way using the "filament" plug-in in Imaris 3D interactive visualization software (Bitplane, Zurich, Switzerland). To show the colocalization of two interest proteins or the location of proteins in microglia, any immunostaining signals outside of Iba-1staining area were masked using "surface" and "mask" plug-in in Imaris software, and each protein was made into the surface according to each staining signal. Then, final $3 \mathrm{D}$ rendering surface images were made by combining each protein surface to make a representative image.

Brain fractionation. Frozen brains (one hemisphere each animal) were homogenized with Dounce homogenizer in $2 \mathrm{ml}$ of sucrose buffer $(0.32 \mathrm{M}$ Sucrose in 50 $\mathrm{mM}$ Tris- $\mathrm{HCl}, \mathrm{pH}$ 7.4) added with Halt ${ }^{\mathrm{Tw}}$ Protease and Phosphatase Inhibitor Cocktail (\#78440, Thermofisher Scientific), and spun-down for $10 \mathrm{~min}$ at $1000 \times g$ $4{ }^{\circ} \mathrm{C}$. The supernatant $(\sim 1.3 \mathrm{ml})$ was collected and $0.9 \mathrm{ml}$ of lysates were mixed with $0.1 \mathrm{ml}$ of $10 \%$ Triton X-100 to make $1 \%$ Triton X-100 in sucrose buffer. After spindown for $10 \mathrm{~min}$ at $16,000 \times g 4^{\circ} \mathrm{C}$, the supernatant was collected into the new tube (detergent-soluble fraction), and the pellet was washed with $1 \%$ Triton X-100 two times to remove any soluble proteins. Finally, the washed pellet was resuspended in $0.1 \mathrm{ml}$ of $1 \% \mathrm{SDS} / 1 \%$ Triton X-100/sucrose buffer and sonicated with the probe-tip sonicator in level ' 2 ' for $10 \mathrm{~s}$ (Fisher Scientific 550 Sonic Dismembrator). After spin-down for $5 \mathrm{~min}$ at $16,000 \times g \mathrm{RT}$, the supernatant was collected into the new tube (detergent-insoluble fraction). Protein concentration was determined by Pierce BCA Protein Assay Kit (\#23225, Thermofisher Scientific).

Immunoprecipitation. Brain lysate from 1\% Triton X-100/sucrose buffer fractions or cultured microglia lysed in $1 \%$ Triton X-100 PBS were incubated with asynuclein antibody (clone $2 \mathrm{f} 12$, MABN1817, Millipore) in $4{ }^{\circ} \mathrm{C}$ overnight followed by Dynabeads ${ }^{\text {Tx }}$ Protein G (\#10009D, Thermofisher Scientific). After washing with $1 \%$ Triton X-100 PBS three times, proteins that bound to beads were released by boiling in LDS sample buffer (Thermofisher Scientific) at $95^{\circ} \mathrm{C}$ for $10 \mathrm{~min}$.

Western blot analysis. Cultured cells were lysed on ice in RIPA buffer (50 mM Tris- $\mathrm{HCl}$ (pH 7.4), 1\% NP-40, $1 \mathrm{mM} \mathrm{NaF}, 0.25 \% \mathrm{Na}$-deoxycholate, $1 \mathrm{mM} \mathrm{Na}_{3} \mathrm{VO}_{4}$ and $150 \mathrm{mM} \mathrm{NaCl}$ ) containing Halt ${ }^{\mathrm{TM}}$ protease and phosphatase inhibitor cocktail (\#78440, Thermofisher Scientific). For FACS-sorted microglia, cells were lysed in
$1 \%$ Triton/8 M Urea/PBS buffer to maximize the yield, sonicated with the probe-tip sonicator in level "2" for $10 \mathrm{~s}$ (Fisher Scientific 550 Sonic Dismembrator). For AAV model, total $\sim 30,000$ microglia sorted from FACS were used and, for transgenic mice, $\sim 120,000$ cells were used for each group. Lysates were centrifuged, and proteins in the supernatant were separated by NuPAGE ${ }^{\circledast}$ Precast Gel System (Thermofisher Scientific). Membranes were incubated with primary antibodies, including actin (\#3700 S, Cell Signaling, 1:50000), ATG7 (MAB6608, R\&D, 1:1000), ATG14 (PD026, MBL, 1:1000), dopamine transporter (MAB369, Millipore, 1:1000), ERK1/2 (\#9107S, Cell Signaling, 1:1000), p-ERK1/2 (Thr202/Tyr204, \#4370P, Cell Signaling, 1:1000), GFP/YFP (MA5-15256, Thermofisher Scientific, 1:2000), IкB (\#4814, Cell Signaling, 1:1000), p-IRF3 (S396, \#29047, Cell Signaling, 1:1000), IRF3 (\#4302, Cell Signaling, 1:1000), JNK (\#9252S, Cell Signaling, 1:1000) p-JNK (Thr183/Tyr185, \#4671S, Cell Signaling, 1:1000), LC3B (\#2775S, Cell Signaling, 1:1000), NBR1 (16004-1-AP, Proteintech, 1:1000), NDP52 (12229-1-AP, Proteintech, 1:1000), NQO-1 (11451-1-AP, Proteintech, 1:1000), HO-1 (sc-10789, Santa Cruz, 1:1000) p-NF-kB (S536, \#3033S, Cell Signaling, 1:1000), Optineurin (10837-1-AP, Proteintech, 1:1000), p38 (\#9212S, Cell Signaling, 1:1000), p-p38 (Thr180/Tyr182, \#4511S, Cell Signaling, 1:1000), p62 (PM066, MBL, 1:50000), p62 (18420-1-AP, Proteintech, 1:1000), a-synuclein (clone MJFR1, ab138501, Abcam, 1:2000; clone 2f12, MABN1817, Millipore, 1:2000; clone 42, 610787 BD Bioscience, 1:1000; clone D37A6, \# 4179, Cell Signaling, 1:1000), or Tyrosine hydroxylase (T2928, Sigma, 1:2000) at $4^{\circ} \mathrm{C}$ overnight, washed with Tris-buffered saline containing $0.1 \%$ Tween 20 , incubated with secondary antibodies, and visualized with SuperSignal $^{\text {Ts }}$ West Pico Chemiluminescent Substrate (Thermofisher Scientific). Uncropped scan images for the main figures are presented in Supplementary Fig. 8.

HEK293T-NF-кB-luciferase assay system. HEK293T cells were seeded into a 96well plate at a density of $2 \times 10^{4}$ cells per well overnight. Cells were transfected using Lipofectamine 2000 (Invitrogen) with pcDNA3 vectors encoding either TLR1 (Addgene \#13014), TLR2 (Addgene \#13015), TLR4 (Addgene \#13018), MD2 (Addgene \#13028), CD14 (Addgene \#13645), TLR5 (Addgene \#13019), or TLR6 (Addgene \#13020) with pGL4.32[luc2P/NF-kB-RE/Hygro] vector containing five copies of an NF- $\kappa \mathrm{B}$ response element (NF- $\kappa \mathrm{B}-\mathrm{RE}$ ) that drives transcription of the luciferase reporter gene luc2P (Photinus pyralis) (\#N111A, Promega, Madison, WI). Vectors were gifts from the Doug Golenbock Lab through Addgene. After $24 \mathrm{~h}$, cells were treated with either EndoClear ${ }^{\mathrm{TM}}$ human recombinant $\alpha$-synuclein (AS5555, Anaspec, CA), lipopolysaccharide (LPS, \#L7770, Sigma-Aldrich), $\mathrm{Pam}_{3} \mathrm{CSK}_{4}$ (\#tlrl-pms, Invivogen, CA), or recombinant Flagellin (Rec FLA-ST, \#tlrl-flic-10, Invivogen, CA). Following $24 \mathrm{~h}$ of stimulation by the ligand, the luciferase activity was measured using the ONE-Glo ${ }^{\mathrm{TM}}$ Luciferase assay system (\#E6110, Promega, Madison, WI).

Electron microscope. Cell cultures grown on Permanox slides (Electron Microscopy Sciences (EMS), Hatfield, PA) were taken from incubation, and directly placed in 2\% glutaraldehyde (EMS) and 2\% paraformaldehyde (EMS)/0.1 M sodium cacodylate buffer (EMS) for a minimum of $2 \mathrm{~h}$ at $4{ }^{\circ} \mathrm{C}$. Cells were washed, fixed in $1 \%$ aqueous osmium tetroxide at RT for $1 \mathrm{~h}$, washed, and transferred to $2 \%$ aqueous uranyl acetate at RT for $1 \mathrm{~h}$. Slides were washed with distilled water, dehydrated in an ascending aqueous ethanol series, and then embedded in Epon resin (EMS). Inverted BEEM capsules (\#3, EMS) were placed directly over regions of interest, filled with fresh resin, and transferred to a vacuum oven for heat polymerization at $60^{\circ} \mathrm{C}$ for $12-24 \mathrm{~h}$. To separate the cells from the slides, a hot plate was heated to $60^{\circ} \mathrm{C}$, and the slides placed directly on a pre-heated hot plate for exactly $3 \mathrm{~min}$ and $30 \mathrm{~s}$. The capsules were removed from the hot plate, and carefully dislodged free from the slide using a plier. Ultrathin $(85 \mathrm{~nm})$ sections were collected onto 300 mesh copper grids (EMS) using a Leica UC7 ultramicrotome (Leica Biosystems Inc., Buffalo Grove, IL), contrast stained with uranyl acetate and lead citrate, and imaged on a Hitachi 7700 transmission electron microscope (Hitachi High Technologies America, Inc., Dallas, TX) equipped with an AMT 2 K x $2 \mathrm{~K}$ digital camera (Advanced Microscopy Techniques, Corp., Woburn, MA).

Image analysis and quantification. For counting p62-positive or p62/a-synuclein-positive puncta in microglia in vivo, fixed brain slices were stained with primary antibodies against human $\alpha$-synuclein, p62, and Iba- 1 and $z$-stack pictures were obtained with $\times 63$ magnification $(1024 \times 1024$ pixels $)$ at multiple areas of the striatum using a confocal microscope (Zeiss LSM 780, Carl Zeiss). Puncta were counted inside of Iba-1-positive area in every single focal plane throughout the whole $z$-stack manually. In cultured cells treated with EndoClear ${ }^{\mathrm{TM}}$ human $\alpha$ synuclein (AS5555, Anaspec, CA), pictures were taken with $\times 20$ magnification from multiple areas (up to 15 areas per coverslip), and ubiquitin/a-synucleinpositive puncta were counted by a plug-in "Cell counter" in Fiji software (National Institutes of Health, MD). Then, the number of puncta each picture was divided by cell number and normalized to each control group. For quantification of p-S129 asynuclein $\mathrm{DAB}$ staining, pictures were taken with $\times 20$ magnification, and positive signals were counted by a semi-automated way by using "Find Maxima" in Fiji software (National Institutes of Health, MD) after setting the threshold. Values were normalized to the average values from the control group in each cohort. For stereology counting of TH-positive or Nissl-stained cell bodies in the SNpc, one in every six sections was selected with a random start, and a total of five brain slices 
on average were used for each mouse for IHC labeling for $\mathrm{TH}$, including DAB enhancement, followed by cresyl violet staining to reveal all neurons. A Zeiss Axioplan 2 was used for tissue slice imaging with a $\times 20$ objective, and Stereo Investigator was used to estimating the total number of neurons in the region of interest using the following parameters: frame sizes, $150 \times 150 \mu \mathrm{m}$; grid sizes, $250 \times$ $250 \mu \mathrm{m}$; top guard zone height, $2 \mu \mathrm{m}$; and optical dissector height, $8 \mu \mathrm{m}$. These parameters yielded a coefficient of error $<10 \%$ throughout the analysis.

Statistics and reproducibility. The statistical significance of differences between two groups was determined using the unpaired two-tailed Student's $t$ test or Mann-Whitney $U$ test based on the normality test. For multiple-means comparisons, statistical significance was determined by one-way analysis of variance followed by Newman-Keuls post hoc test or two-way analysis of variance with Bonferroni post hoc test using GraphPad Prism 5 (GraphPad Software, CA, USA). All values are reported as mean \pm SEM. Data are representative of at least three independent experiments unless indicated. The source data underlying Figs. 1b, c, g, 2c, f, g, i, 3a, b, e, g, h, 4a-e, g, h, j, k, 5a-i, 6e, f, h, j, 7b, d, and Supplementary Fig. 1e, f, $4 b, d-j, 5 b, e, 6 a-c, 7 b, d, e, g, i$ are provided as a Source Data file.

Reporting summary. Further information on research design is available in the Nature Research Reporting Summary linked to this article.

\section{Data availability}

The authors declare that all data supporting the findings of this study are available within the paper and its supplementary information files.

Received: 16 January 2020; Accepted: 17 February 2020;

Published online: 13 March 2020

\section{References}

1. Iwai, A. et al. The precursor protein of non-A beta component of Alzheimer's disease amyloid is a presynaptic protein of the central nervous system. Neuron 14, 467-475 (1995).

2. Bendor, J. T., Logan, T. P. \& Edwards, R. H. The function of alpha-synuclein. Neuron 79, 1044-1066 (2013).

3. Chandra, S., Gallardo, G., Fernandez-Chacon, R., Schluter, O. M. \& Sudhof, T. C. Alpha-synuclein cooperates with CSPalpha in preventing neurodegeneration. Cell 123, 383-396 (2005).

4. Murphy, D. D., Rueter, S. M., Trojanowski, J. Q. \& Lee, V. M. Synucleins are developmentally expressed, and alpha-synuclein regulates the size of the presynaptic vesicular pool in primary hippocampal neurons. J. Neurosci. 20, 3214-3220 (2000).

5. Baba, M. et al. Aggregation of alpha-synuclein in Lewy bodies of sporadic Parkinson's disease and dementia with Lewy bodies. Am. J. Pathol. 152, 879-884 (1998).

6. Toledo, J. B. et al. Pathological alpha-synuclein distribution in subjects with coincident Alzheimer's and Lewy body pathology. Acta Neuropathol. 131, 393-409 (2016).

7. Chartier-Harlin, M. C. et al. alpha-synuclein locus duplication as a cause of familial Parkinson's disease. Lancet 364, 1167-1169 (2004).

8. Singleton, A. B. et al. alpha-synuclein locus triplication causes Parkinson's disease. Science 302, 841-841 (2003).

9. Kordower, J. H., Chu, Y., Hauser, R. A., Freeman, T. B. \& Olanow, C. W. Lewy body-like pathology in long-term embryonic nigral transplants in Parkinson's disease. Nat. Med. 14, 504-506 (2008).

10. Li, J. Y. et al. Lewy bodies in grafted neurons in subjects with Parkinson's disease suggest host-to-graft disease propagation. Nat. Med. 14, 501-503 (2008).

11. Emmanouilidou, E. et al. Assessment of alpha-synuclein secretion in mouse and human brain parenchyma. PLoS One 6, e22225 (2011).

12. Jang, A. et al. Non-classical exocytosis of alpha-synuclein is sensitive to folding states and promoted under stress conditions. J. Neurochem. 113, 1263-1274 (2010).

13. Yamada, K. \& Iwatsubo, T. Extracellular alpha-synuclein levels are regulated by neuronal activity. Mol. Neurodegener. 13, 9 (2018).

14. Luk, K. C. et al. Pathological alpha-synuclein transmission initiates Parkinsonlike neurodegeneration in nontransgenic mice. Science 338, 949-953 (2012).

15. Braak, H. et al. Staging of brain pathology related to sporadic Parkinson's disease. Neurobiol. Aging 24, 197-211 (2003).

16. Lee, H. J., Suk, J. E., Bae, E. J. \& Lee, S. J. Clearance and deposition of extracellular alpha-synuclein aggregates in microglia. Biochem. Biophys. Res. Commun. 372, 423-428 (2008).

17. Lee, H. J. et al. Direct transfer of alpha-synuclein from neuron to astroglia causes inflammatory responses in synucleinopathies. J. Biol. Chem. 285 , 9262-9272 (2010).
18. Mao, X. et al. Pathological alpha-synuclein transmission initiated by binding lymphocyte-activation gene 3. Science 353, aah3374 (2016).

19. Kim, C. et al. Neuron-released oligomeric alpha-synuclein is an endogenous agonist of TLR2 for paracrine activation of microglia. Nat. Commun. 4, 1562 (2013).

20. Cuervo, A. M., Stefanis, L., Fredenburg, R., Lansbury, P. T. \& Sulzer, D. Impaired degradation of mutant alpha-synuclein by chaperone-mediated autophagy. Science 305, 1292-1295 (2004).

21. Tofaris, G. K. et al. Ubiquitin ligase Nedd4 promotes alpha-synuclein degradation by the endosomal-lysosomal pathway. Proc. Natl Acad. Sci. USA 108, 17004-17009 (2011).

22. Vogiatzi, T., Xilouri, M., Vekrellis, K. \& Stefanis, L. Wild type alpha-synuclein is degraded by chaperone-mediated autophagy and macroautophagy in neuronal cells. J. Biol. Chem. 283, $23542-23556$ (2008).

23. Webb, J. L., Ravikumar, B., Atkins, J., Skepper, J. N. \& Rubinsztein, D. C. alpha-synuclein is degraded by both autophagy and the proteasome. J. Biol. Chem. 278, 25009-25013 (2003)

24. Lin, Y. C., Kuo, H. C., Wang, J. S. \& Lin, W. W. Regulation of inflammatory response by 3-methyladenine involves the coordinative actions on akt and glycogen synthase kinase 3 beta rather than autophagy. J. Immunol. 189 , 4154-4164 (2012).

25. $\mathrm{Wu}, \mathrm{Y}$. T. et al. Dual role of 3-methyladenine in modulation of autophagy via different temporal patterns of inhibition on class I and III phosphoinositide 3kinase. J. Biol. Chem. 285, 10850-10861 (2010).

26. Mizushima, N., Yoshimori, T. \& Ohsumi, Y. The role of Atg proteins in autophagosome formation. Annu. Rev. Cell Dev. Biol. 27, 107-132 (2011)

27. Yamamoto, A. \& Yue, Z. Autophagy and its normal and pathogenic states in the brain. Annu. Rev. Neurosci. 37, 55-78 (2014).

28. Stolz, A., Ernst, A. \& Dikic, I. Cargo recognition and trafficking in selective autophagy. Nat. Cell Biol. 16, 495-501 (2014).

29. Oliveras-Salva, M. et al. rAAV2/7 vector-mediated overexpression of alphasynuclein in mouse substantia nigra induces protein aggregation and progressive dose-dependent neurodegeneration. Mol. Neurodegener. 8, 44 (2013).

30. Keren-Shaul, H. et al. A unique microglia type associated with restricting development of Alzheimer's disease. Cell 169, 1276 (2017).

31. Krasemann, S. et al. The TREM2-APOE pathway drives the transcriptional phenotype of dysfunctional microglia in neurodegenerative diseases. Immunity 47, 566 (2017).

32. Butovsky, O. et al. Identification of a unique TGF-beta dependent molecular and functional signature in microglia. Nat. Neurosci. 17, 131-143 (2014).

33. Parkhurst, C. N. et al. Microglia promote learning-dependent synapse formation through brain-derived neurotrophic factor. Cell 155, 1596-1609 (2013).

34. Lamark, T., Kirkin, V., Dikic, I. \& Johansen, T. NBR1 and p62 as cargo receptors for selective autophagy of ubiquitinated targets. Cell Cycle $\mathbf{8}$, 1986-1990 (2009).

35. Mizushima, N., Yamamoto, A., Matsui, M., Yoshimori, T. \& Ohsumi, Y. In vivo analysis of autophagy in response to nutrient starvation using transgenic mice expressing a fluorescent autophagosome marker. Mol. Biol. Cell 15, 1101-1111 (2004).

36. Ronan, B. et al. A highly potent and selective Vps34 inhibitor alters vesicle trafficking and autophagy. Nat. Chem. Biol. 10, 1013 (2014).

37. Liu, Q. S. et al. Discovery of 1-(4-(4-Propionylpiperazin-1-yl)-3(trifluoromethyl)phenyl)-9-(quinolin-3-yl)benzo[h][1,6]naphthyridin-2(1H)one as a highly potent, selective mammalian target of rapamycin (mTOR) inhibitor for the treatment of cancer. J. Med. Chem. 53, 7146-7155 (2010).

38. Komatsu, M. et al. Impairment of starvation-induced and constitutive autophagy in Atg7-deficient mice. J. Cell Biol. 169, 425-434 (2005).

39. Matsunaga, K. et al. Autophagy requires endoplasmic reticulum targeting of the PI3-kinase complex via Atg14L. J. Cell Biol. 190, 511-521 (2010).

40. Fellner, L. et al. Toll-like receptor 4 is required for alpha-synuclein dependent activation of microglia and astroglia. Glia 61, 349-360 (2013).

41. Rannikko, E. H., Weber, S. S. \& Kahle, P. J. Exogenous alpha-synuclein induces toll-like receptor 4 dependent inflammatory responses in astrocytes. BMC Neurosci. 16, 57 (2015).

42. Matsunaga, N., Tsuchimori, N., Matsumoto, T. \& Ii, M. TAK-242 (resatorvid), a small-molecule inhibitor of Toll-like receptor (TLR) 4 signaling, binds selectively to TLR4 and interferes with interactions between TLR4 and its adaptor molecules. Mol. Pharm. 79, 34-41 (2011).

43. Sakaguchi, S. et al. Essential role of IRF-3 in lipopolysaccharide-induced interferon-beta gene expression and endotoxin shock. Biochem. Bioph. Res. Commun. 306, 860-866 (2003).

44. Zanoni, I. et al. CD14 controls the LPS-induced endocytosis of Toll-like receptor 4. Cell 147, 868-880 (2011).

45. Zhong, Z. Y. et al. NF-kappa B restricts inflammasome activation via elimination of damaged mitochondria. Cell 164, 896-910 (2016) 
46. Fujita, K. \& Srinivasula, S. M. TLR4-mediated autophagy in macrophages is a p62-dependent type of selective autophagy of aggresome-like induced structures (ALIS). Autophagy 7, 552-554 (2011).

47. Settembre, C. et al. TFEB links autophagy to lysosomal biogenesis. Science 332, 1429-1433 (2011).

48. Gay, N. J., Symmons, M. F., Gangloff, M. \& Bryant, C. E. Assembly and localization of Toll-like receptor signalling complexes. Nat. Rev. Immunol. 14, 546-558 (2014).

49. Friedman, L. G. et al. Disrupted autophagy leads to dopaminergic axon and dendrite degeneration and promotes presynaptic accumulation of alpha-synuclein and LRRK2 in the brain. J. Neurosci. 32, 7585-7593 (2012).

50. Komatsu, M. et al. Loss of autophagy in the central nervous system causes neurodegeneration in mice. Nature 441, 880-884 (2006).

51. Redmann, M., Wani, W. Y., Volpicelli-Daley, L., Darley-Usmar, V. \& Zhang, J. Trehalose does not improve neuronal survival on exposure to alpha-synuclein pre-formed fibrils. Redox Biol. 11, 429-437 (2017).

52. Shi, C. S. \& Kehrl, J. H. MyD88 and Trif target Beclin 1 to trigger autophagy in macrophages. J. Biol. Chem. 283, 33175-33182 (2008).

53. Watson, R. O., Manzanillo, P. S. \& Cox, J. S. Extracellular M. tuberculosis DNA targets bacteria for autophagy by activating the host DNA-sensing pathway. Cell 150, 803-815 (2012).

54. Xu, Y. et al. Toll-like receptor 4 is a sensor for autophagy associated with innate immunity. Immunity 27, 135-144 (2007).

55. Mostowy, S. et al. p62 and NDP52 proteins target intracytosolic Shigella and Listeria to different autophagy pathways. J. Biol. Chem. 286, 26987-26995 (2011).

56. Stefanova, N. et al. Toll-like receptor 4 promotes alpha-synuclein clearance and survival of nigral dopaminergic neurons. Am. J. Pathol. 179, 954-963 (2011).

57. Dzamko, N. et al. Toll-like receptor 2 is increased in neurons in Parkinson's disease brain and may contribute to alpha-synuclein pathology. Acta Neuropathol. 133, 303-319 (2017).

58. Kim, C. et al. Antagonizing neuronal Toll-like receptor 2 prevents synucleinopathy by activating autophagy. Cell Rep. 13, 771-782 (2015).

59. Main, B. S. et al. Type-1 interferons contribute to the neuroinflammatory response and disease progression of the MPTP mouse model of Parkinson's disease. Glia 64, 1590-1604 (2016).

60. Martinez, J. et al. Molecular characterization of LC3-associated phagocytosis reveals distinct roles for Rubicon, NOX2 and autophagy proteins. Nat. Cell Biol. 17, 893-906 (2015).

61. Ahn, K. J., Paik, S. R., Chung, K. C. \& Kim, J. Amino acid sequence motifs and mechanistic features of the membrane translocation of alpha-synuclein. $J$. Neurochem. 97, 265-279 (2006).

62. Lee, H. J. et al. Assembly-dependent endocytosis and clearance of extracellular alpha-synuclein. Int. J. Biochem. Cell Biol. 40, 1835-1849 (2008).

63. Park, J. Y. et al. On the mechanism of internalization of alpha-synuclein into microglia: roles of ganglioside GM1 and lipid raft. J. Neurochem. 110, 400-411 (2009).

64. Chang, D. et al. A meta-analysis of genome-wide association studies identifies 17 new Parkinson's disease risk loci. Nat. Genet. 49, 1511-1516 (2017).

65. Gan-Or, Z., Dion, P. A. \& Rouleau, G. A. Genetic perspective on the role of the autophagy-lysosome pathway in Parkinson disease. Autophagy 11, 1443-1457 (2015).

66. Komatsu, M. et al. Homeostatic levels of p62 control cytoplasmic inclusion body formation in autophagy-deficient mice. Cell 131, 1149-1163 (2007).

67. Choi, J. et al. The parasitophorous vacuole membrane of toxoplasma gondii is targeted for disruption by ubiquitin-like conjugation systems of autophagy. Immunity 40, 924-935 (2014).
68. Rockenstein, E. et al. Differential neuropathological alterations in transgenic mice expressing alpha-synuclein from the platelet-derived growth factor and Thy-1 promoters. J. Neurosci. Res. 68, 568-578 (2002).

69. Goodwin, L. O. et al. Large-scale discovery of mouse transgenic integration sites reveals frequent structural variation and insertional mutagenesis. Genome Res. 29, 494-505 (2019).

70. Veremeyko, T., Starossom, S. C., Weiner, H. L. \& Ponomarev, E. D. Detection of MicroRNAs in microglia by real-time PCR in normal CNS and during neuroinflammation. J. Vis. Exp. 65, e4097 (2012).

\section{Acknowledgements}

This work was supported by an NIH grant (RO1, NS060123, and P50NS094733). We thank the assistance of members in the core facilities for Flow Cytometry, Microscopy, and qPCR at the Icahn School of Medicine at Mount Sinai. We thank NeuroScience Associates (Michael J. Fox Foundation) for the Thy-1-SNCA animal information and experiment. We are grateful to all members of Yue laboratory at the Icahn School of Medicine at Mount Sinai.

\section{Author contributions}

Z.Y. conceived the project, supervised the entire study, and wrote the paper; I.C. designed, performed, and analyzed most of the experiments and wrote the paper; Y.Z. helped for the stereotaxic injection and stereological counting; S.S. performed NF- $\mathrm{B}$ luciferase experiments; M.P. helped in vitro results and analysis; Q.W. and B.Z. helped for analyzing the data; K.P. helped for preparing mice and writing the paper.

\section{Competing interests}

The authors declare no competing interests.

\section{Additional information}

Supplementary information is available for this paper at https://doi.org/10.1038/s41467 020-15119-w.

Correspondence and requests for materials should be addressed to Z.Y.

Peer review information Nature Communications thanks the anonymous reviewer(s) for their contribution to the peer review of this work.

Reprints and permission information is available at http://www.nature.com/reprints

Publisher's note Springer Nature remains neutral with regard to jurisdictional claims in published maps and institutional affiliations.

Open Access This article is licensed under a Creative Commons Attribution 4.0 International License, which permits use, sharing, adaptation, distribution and reproduction in any medium or format, as long as you give appropriate credit to the original author(s) and the source, provide a link to the Creative Commons license, and indicate if changes were made. The images or other third party material in this article are included in the article's Creative Commons license, unless indicated otherwise in a credit line to the material. If material is not included in the article's Creative Commons license and your intended use is not permitted by statutory regulation or exceeds the permitted use, you will need to obtain permission directly from the copyright holder. To view a copy of this license, visit http://creativecommons.org/ licenses/by/4.0/

(C) The Author(s) 2020 